Rev. Est. de Políticas Públicas, 5(2): julio 2019 - noviembre 2019, 71-100

http://dx.doi.org/10.5354/0719-6296.2019.53803

ISSN edición web: 0719-6296

(C) Copyright 2019: Universidad de Chile, Santiago (Chile)

\title{
Cambios en la tasa social de descuento y su impacto en la tasa de aprobación de proyectos de agua potable rural recomendados para su ejecución
}

\author{
Nelson Cabrera \\ Investigador Independiente
}

\section{Resumen}

Este trabajo revisa los aspectos teóricos asociados a los enfoques y metodologías existentes para determinar el valor de la Tasa Social de Descuento y las implicancias en el cambio de su valor en los proyectos de Agua Potable Rural en Chile. Se estiman los niveles de Elasticidad en la Tasa de Aprobación de Proyectos y se evalúan en un contexto de tasa de descuento en disminución, tal como se han registrado estas dos últimas décadas. Los resultados sugieren la no presencia de niveles de elasticidad significativos en la Tasa de Aprobación de los Proyectos. El análisis es realizado considerando el uso del factor de descuento exponencial, tradicionalmente utilizado por la teoría económica para la evaluación de proyectos, como así también el uso de factores de descuentos basados en tasas de descuentos decrecientes (hiperbólicas). Se utilizó en el estudio una muestra de 42 proyectos de Agua Potable Rural pertenecientes a las provincias de la Región del Bio Bio.

Palabras clave: Tasa Social de Descuento, Elasticidad Tasa de Aprobación Proyectos, Políticas Públicas.

Changes in the social discount rate and its impact on the approval rate of rural drinking water projects recommended for execution

\begin{abstract}
This paper reviews the theoretical aspects associated with the existing approaches and methodologies to determine the value of the Social Discount Rate and the implications for changing its value in Rural Potable Water projects in Chile. The levels of Elasticity in the Project Approval Rate are estimated and evaluated in a context of decreasing discount rate, as recorded in the last two decades. The results suggest the absence of significant levels of elasticity in the Project Approval Rate. The analysis is performed considering the use of the exponential discount factor, traditionally used by economic theory for the evaluation of projects, as well as the use of discount factors based on decreasing (hyperbolic) discount rates. A sample of 42 Rural Drinking Water projects belonging to the provinces of the Bio Bio Region was used in the study.
\end{abstract}

Keywords: Discount Social Rate, Elasticity Approval Rate Projects, Public Policies.

*Dirección de correspondencia [Correspondence address]:

Nelson Cabrera, Investigador independiente

E-mail: nelsoncabrerat@hotmail.com 


\section{Introducción}

La Tasa Social de Descuento (TSD) representa el costo de oportunidad de la sociedad respecto de las decisiones de consumo e inversión que toma el Estado, por lo que su valor juega un papel importante en los resultados económicos del país. Los distintos enfoques económicos utilizados para determinar el valor de la TSD dependen en buena parte de las características de las economías, donde las industrializadas se destacan por preferencias de la sociedad por decisiones basadas en el consumo y las economías en desarrollo por decisiones de inversión.

En consideración a lo anterior, esta investigación se centra en la importancia que toma el valor de la TSD y su impacto en un sector de la economía como es el suministro del Agua Potable Rural (APR) en Chile. Este sector económico surgido a principios de los años sesenta fundamentalmente por iniciativas de organismos internacionales como el Banco Interamericano de Desarrollo (BID) e implementado en el país por medio del "Programa APR" de la Dirección de Obras Hidráulicas (DOH), proporcionan infraestructura a la población rural del país basado en sistemas de agua potable. ${ }^{1}$

Por su parte, el nuevo marco regulatorio que afectará al sector, según la Ley 20.998 aprobada el año 2017 y que entrará en vigencia una vez aprobado el reglamento, ${ }^{2}$ trae consigo cambios significativos en la estructura de funcionamiento del modelo de gestión asociada al programa APR, el que no ha experimentado cambios significativos desde su implementación a principios de los años sesenta. Lo anterior, destaca el rol de la TSD en la asignación de los recursos destinados a este sector bajo esta nueva normativa y la importancia de su estudio para dimensionar los impactos en los resultados del programa.

En consideración a ello, este trabajo se estructura de la manera que se indica a continuación. En la sección 2 se indican las bases teóricas y conceptuales relativas a los principales enfoques para determinar el valor de la TSD y se realiza una descripción de los proyectos APR implementados en Chile. En la

\footnotetext{
${ }^{1}$ En base al documento "Metodología de Formulación y Evaluación de Proyectos de Agua Potable Rural" del Ministerio de Desarrollo Social (MDS), se define al Sistema APR como al conjunto de obras de captación, tratamiento, conducción, regulación, distribución y suministro intradomiciliario de agua potable, y que corresponde a la solución adoptada para las localidades rurales concentradas.

${ }^{2}$ Finalizado este estudio, el reglamento se encontraba en trámite de toma de razón por parte de la Contraloría General de la República, Chile.
}

sección 3 se indica la metodología utilizada para el análisis económico efectuado a los proyectos APR, considerando el enfoque tradicional de TSD y enfoques basados en el uso de tasas de descuentos decrecientes. Se indica además el procedimiento utilizado para estimar la Tasa de Descuento Límite de Aprobación (TDLA) de los proyectos APR y los valores de elasticidad de la función Tasa de Aprobación de Proyectos (TAP). En la sección 4 se analizan los resultados obtenidos en la TAP y la elasticidad de la función TAP. Además, se realiza un análisis de estabilidad de los datos de los 42 proyectos APR y se evalúan los impactos en la TAP al considerar políticas públicas que buscan dar solución a los principales problemas detectados por la DOH al Programa APR. En la sección 5, se entregan las principales conclusiones del estudio.

\section{Bases teóricas y conceptuales}

\subsection{Tasa Social de Descuento}

Si bien la teoría económica no ha logrado un consenso respecto de que enfoque utilizar para la determinación de la tasa de descuento que mejor se ajuste a las decisiones de los agentes económicos, se sugieren dos enfoques para determinar su valor. El primero tienen su origen en decisiones que se basan en el consumo, representado por la Tasa Social de Preferencia Intertemporal (TSPI) y el segundo se basa en las decisiones de inversión, representado por la tasa Costo de Oportunidad Social del Capital (COSC).

La TSPI sugiere que la TSD corresponde a aquella tasa en la cual las preferencias del conjunto de la sociedad son por consumo presente por sobre consumo futuro (Correa, 2006: 93). En lo fundamental esta tasa refleja las expectativas del aumento en el consumo que la sociedad tiene del futuro, a cambio de retrasar el consumo presente. Por lo que su utilización como TSD se basa en que la implementación de los proyectos públicos desplaza el consumo corriente. Por su parte, la tasa COSC se concentra en el hecho de que los proyectos públicos desplazan a las inversiones privadas, por lo que el rendimiento esperado de un proyecto público debiera por lo menos coincidir con el costo de oportunidad de los fondos, el cual sería igual al rendimiento esperado de la inversión en el sector privado. ${ }^{3}$

\footnotetext{
${ }^{3}$ En base al documento "Metodología de Formulación y Evaluación de Proyectos de Agua Potable Rural" del Ministerio de Desarrollo Social (MDS), se define al Sistema APR como al conjunto de obras de captación, tratamiento, conduc-
} 
En una economía cerrada y sin distorsiones, ambos enfoques debieran coincidir en un mismo valor y reflejar de este modo la TSD. ${ }^{4}$ Sin embargo, esto no ocurre, lo cual trae implicancia en las decisiones de ahorro e inversión que son realizadas por los agentes económicos, toda vez la tasa de interés de mercado no será igual a la TSD, lo que se traduce en que el valor actual de los beneficios sociales netos (VABN) de los proyectos que son implementados desde el punto de vista país, no serán iguales al VABN de los proyectos que son realizados por los privados.

En Chile la metodología utilizada para determinar la TSD, ${ }^{5}$ como así también en buena parte de los países en desarrollo y organismos multilaterales de fomento al desarrollo ${ }^{6}$ (ver trabajos de Oddene, 2011; Campos et al., 2015 y 2016) recogen en parte lo propuesto por los dos principales enfoques. Esta metodología fue desarrollada por Harberger (1972) y considera la utilización de los criterios de eficiencia de Pareto. Su valor se determina en base a la valoración alternativa que realiza la sociedad por el uso de los fondos de inversión, que pueden venir del ahorro privado, la inversión privada y ahorro externo.

La TSD desarrollada por la metodología Harberger es representada, según los estudios realizados por Cartes et al. (2004), por la siguiente fórmula:

$$
T S D=(\beta * t p)+(\theta * q)+(\alpha * C M g x)
$$

Donde: $\beta, \theta, \mathrm{y} \alpha$ son los ponderadores de estos valores, los cuales reflejan el impacto relativo que produce el uso de cada una de las fuentes de financiamiento.

$t p, q$, y $C m g x$ son los costos que la sociedad percibe, asociadas al ahorro privado, al rendimiento de la inversión y al endeudamiento externo, respectivamente.

ción, regulación, distribución y suministro intradomiciliario de agua potable, y que corresponde a la solución adoptada para las localidades rurales concentradas.

${ }^{4}$ Cartes et al. (2004), indican al mercado de capitales como una de las principales causas que impiden como combinar ambas tasas para obtener la TSD. Por su parte Edwards (2016) menciona como principales distorsiones los impuestos, asimetrías de información y externalidades.

${ }^{5}$ Esta se basa en el estudio "La Tasa Social de Descuento en Chile", que fue encargado por el MDS (Chile) a los académicos Cartes et al. (2004), Facultad de Ingeniería de la Universidad de Chile.

${ }^{6}$ Dentro de los organismos multilaterales destacan: Banco Mundial, Banco Interamericano de Desarrollo (BID) y Banco de Desarrollo de Asia.
Respecto del valor de la TSD en Chile, para el año 2018 el MDS fija su valor en un 6\%. En los últimos 20 años, según se observa en la Figura 1, su valor ha seguido una tendencia decreciente, la cual ha ido en línea con las tasas de interés de los mercados internacionales. $\mathrm{Y}$ en cuanto a la última década, para gran parte de ese periodo, su valor se ha manteniendo constante e igual al $6 \%$.

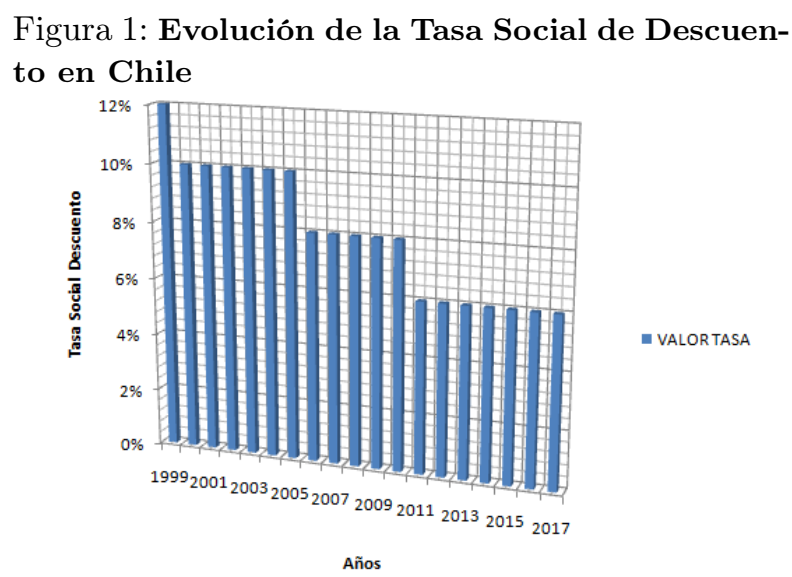

Fuente: Elaboración propia en base información del MDS.

\subsection{Tasas de Descuento Decrecientes}

La teoría económica respecto de las decisiones intertemporales de los individuos, ha planteado tradicionalmente un modelo que represente esa situación, al que ha denominado Modelo de Utilidad Descontada, el cual se basa en los estudios realizados por Samuelson (1937). Este modelo supone que la utilidad (o desutilidad) de los eventos futuros se les aplique un factor de descuento fijo $\delta$, pero con una magnitud declinante $(0<\delta<1)$. La formulación del Modelo de Utilidad Descontada para efectos de su formalización, considera una función exponencial descendente para definir el factor de descuento. Para intervalos de tiempo discretos, este factor queda representado de la siguiente forma:

$$
\delta(X ; t)=\delta^{t}=\frac{1}{(1+X)^{t}}
$$

Donde la variable $\mathrm{X}$ representa la tasa de descuento $\mathrm{y} \mathrm{t}$ los periodos definidos en forma discreta desde $0,1,2,3,4, \ldots$

En los análisis teóricos y empíricos, realizados al Modelo de Utilidad Descontada, la tasa de descuento es independiente del tiempo y garantiza consisten- 
cia dinámica de sus elecciones temporales, esto es, mantiene las preferencias de los agentes invariantes respecto del sólo paso del tiempo (Romano, 2014: 13). Por lo tanto, este modelo para descontar los flujos de beneficios y costos que se generan a lo largo del tiempo, debiera utilizar una TSD de valor constante en el tiempo. Sin embargo, evidencia empírica indica la presencia de violaciones al Modelo de Utilidad Descontada que se manifiestan en que los individuos aplican descuentos más pronunciados a escalas de tiempo menores, como así también la existencia de una preferencia por un sesgo por el presente.

Bajo este contexto, surgen corrientes que proponen modelos que se basan en enfoques de tasas de descuento decrecientes. Uno de estos enfoques considera el uso de tasas de descuento basadas en la función hiperbólica. La representación más básica corresponde al factor de descuento con uso de la función hiperbólica de un parámetro, propuesta en los estudios realizados por el psicólogo Mazur (1987). Con esta función de descuento, el valor presente de un beneficio o costo, se describe de la siguiente manera:

$$
V P=\frac{V F}{(1+X * t)}
$$

Donde $X$ corresponde a la tasas de descuento, $t$ al periodo de tiempo y el factor de descuento en este caso ya no es constante, sino que depende del tiempo y queda definido según se indica en Campos et al. (2015) de la siguiente forma:

$$
\delta(X ; t)=\left[\frac{1+X *(t-1)}{1+X * t}\right]
$$

Una función de descuento con características hiperbólicas, mejora la viabilidad y los resultados de los proyectos de aquellos en los que los costos tienen lugar en los primeros años y los beneficios se encuentran en los últimos años. Por lo tanto, el individuo que decide en base al descuento hiperbólico, está dispuesto a posponer el consumo futuro, por sobre el consumo presente.

Otra alternativa de tasa de descuento decreciente, es aquella que considera individuos que deciden en base a preferencias con características cuasihiperbólicas, como se indican en los estudios de Laibson (1997). Estas aplican tasas de descuento mayores en el corto plazo y menores en el largo plazo, los que son consistentes con los experimentos observados y de este modo representan de mejor forma el comportamiento real de los individuos. ${ }^{7}$

Finalmente, es de importancia destacar que una de las características del enfoque de tasas de descuentos decrecientes y de la cual se diferencia de la función de descuento exponencial, propuesta por el Modelo de Utilidad Descontada, es que tanto la función hiperbólica como la función cuasihiperbólica conducen a decisiones inconsistentes en el tiempo.

\subsection{Agua Potable Rural en Chile}

Antecedentes y análisis descriptivo En el país el suministro de agua potable rural se inicia en el año 1964 por medio del Programa APR, ${ }^{8}$ el cual provee de infraestructura de agua potable rural a localidades concentradas ${ }^{9}$ y semi-concentradas, ${ }^{10}$ cumpliendo con los requisitos establecidos en la metodología de formulación y evaluación de proyectos de agua potable rural del Ministerio de Desarrollo Social (MDS). Para el logro de los objetivos, el programa implementa estrategias por medio de la entrega bienes y servicios los cuales agrupa en tres componentes, ${ }^{11}$ donde el componente 1 corresponde a la ' 'Infraestructura de agua potable entregada a localidades rurales que no cuentan con un servicio APR", el componente 2 se asocia al ' Mejoramiento, ampliación y conservación de infraestructura de APR existente" y el componente 3 correspondientes a recursos destinados a la ' Gestión de los Comités y Cooperativas de APR". ${ }^{12}$

La población objetivo del programa, se enfocaba, inicialmente y hasta el año 2010, sólo en la población rural concentrada, cuando se alcanzó prácticamente plena cobertura de agua potable para dicho sector de la población. ${ }^{13}$ A partir del 2010, se agre-

\footnotetext{
${ }^{7}$ Dentro de los organismos multilaterales destacan: Banco Mundial, Banco Interamericano de Desarrollo (BID) y Banco de Desarrollo de Asia.

${ }^{8} \mathrm{Su}$ ejecución está a cargo de la Dirección de Obras Hidráulicas (DOH), dependiente del Ministerio de Obras Públicas (MOP).

${ }^{9}$ Su ejecución está a cargo de la Dirección de Obras Hidráulicas (DOH), dependiente del Ministerio de Obras Públicas (MOP).

${ }^{10}$ Población constituida por un mínimo de 80 habitantes y con una densidad de al menos 8 viviendas por kilometro de red de agua potable.

${ }^{11}$ Según se indican en el "Informe Final de Evaluación de Infraestructura Hidráulica de Agua Potable Rural" (Dirección de Obras Hidráulicas, 2015), DOH, Chile.

${ }^{12}$ Son responsables de administrar, operar y mantener el sistema de APR.

${ }^{13}$ El Programa habría atendido al $100 \%$ de la población rural concentrada según definición INE/CASEN, alcanzando a más de un millón y medio de habitantes del país.
} 
ga al programa APR la población semi-concentrada que representa la mayor inversión en sistemas nuevos desarrollados. Y a contar del año 2015, se amplía a la población rural dispersa, ${ }^{14}$ abarcando a contar de ello, a toda la población rural.

Según información de la DOH, conforme se indica en Tabla 1, al mes de diciembre del año 2017 existen 1.806 sistemas de APR en el país, alcanzando a 1.682.188 beneficiarios. ${ }^{15}$

La estadística descriptiva para el ítem Número de Arranques y Beneficiarios, de los grupos de sistemas APR instalados a nivel de país, región del Bio Bio y periodo 2010-2017, indican que la distribución de los datos presentan un sesgo positivo, lo cual indica una desviación hacia valores altos, lo que se traduce en una mayor presencia de valores por debajo de la media. Lo anterior, se verifica al aplicar el Test de normalidad ' Shapiro-Wilk", donde se rechazan con un nivel de confianza del $99 \%$ la hipótesis nula que los datos se distribuyen normal.

Además, en la Tabla 1 es posible observar que el $50 \%$ de los sistemas APR instalados en la región del Bio Bio cuentan con al menos 174 arranques, los que abastecen a un número no superior a 712 beneficiarios, valores levemente superiores a los registrados a nivel de país. Sin embargo, los sistemas APR instalados en el periodo 2010-2017, registran un número significativamente inferior a los otros dos grupos, ya que el $50 \%$ de los sistemas APR cuenta al menos con 108 arranques y abastecen a un número no superior a los 428 beneficiarios.

Muestra de proyectos APR El estudio analiza una muestra compuesta por 42 proyectos APR pertenecientes a distintas localidades de la región del Bio Bio, ${ }^{16}$ correspondientes a los procesos de evaluación económica realizados en el periodo 2012-2017. En el Anexo 1 se indica detalle de la muestra, donde se asigna una numeración correlativa al nombre de cada proyecto, partiendo del número 1 hasta el 42. Se destaca que de los 42 proyectos APR, en una primera etapa de evaluación económica no se aprueban un número de 8 de estos proyectos, debido a que no contaban con los requisitos de factibilidad técnicoeconómica, los que tuvieron que pasar a una etapa de reestudio, donde finalmente se recomienda su eje-

\footnotetext{
${ }^{14}$ Población que registra una densidad menor a 8 viviendas por kilometro de red de agua potable.

${ }^{15}$ Población que registra una densidad menor a 8 viviendas por kilometro de red de agua potable.

${ }^{16}$ Esta información fue proporcionada por la DOH, Región del Bio Bio.
}

cución. ${ }^{17}$

Además, es importante mencionar que desde el punto de vista estadístico, si bien la muestra analizada supera un porcentaje de error del $5 \%$, exigida como satisfactorio en un proceso de selección basado en el muestreo aleatorio simple, se reconoce en esta investigación la importancia de la información obtenida de los estamentos gubernamentales respectivos, la que presenta limitaciones debido al número de la población de proyectos APR que sirven de fuente de datos para realizar un análisis probabilístico. ${ }^{18}$

Los datos descriptivos de la muestra se indica en la Tabla 2, donde los ítems Inversión, que se indica en Unidades de Fomento ${ }^{19}(\mathrm{UF}), \mathrm{N}^{\mathrm{O}}$ de Arranques y Beneficiarios; la distribución de los datos presentan un sesgo positivo, lo cual indica una desviación hacia valores altos, lo que se traduce en una mayor presencia de valores por debajo de la media. Lo anterior, se verifica al aplicar el Test de normalidad ' 'Shapiro-Wilk", donde se rechazan con un nivel de confianza del $99 \%$ la hipótesis nula que los datos se distribuyen normal, misma situación presentada en el análisis descriptivo de la población de sistemas APR a nivel de país, región del Bio Bio y periodo 2010-2017.

Por su parte, en la Tabla 3 se indica el análisis comparativo asociados al test de diferencia de medias aplicado a los ítems № Arranques y Beneficiarios, para los grupos de sistemas APR instalados a nivel de país y región del Bio Bio versus la muestra de proyectos APR. ${ }^{20}$ Se observa que no es posible rechazar la hipótesis nula de igualdad de medias de ambos grupos, toda vez el nivel de probabilidad es superior al $5 \%(p>5 \%)$.

Sin embargo, el análisis comparativo al ser realizado respecto de los sistemas APR instalados en el periodo 2010-2017 versus la muestra de proyectos APR; se observan diferencias significativas en los valores medios de ambos grupos, donde se rechaza la hipótesis nula de igualdad de medias para el caso

\footnotetext{
${ }^{17}$ Esta información fue proporcionada por la DOH, Región del Bio Bio.

${ }^{18} \mathrm{Bajo}$ un proceso de muestreo probabilístico, con un error del $5 \%$, el tamaño de la muestra para la región del Bio Bio, debe corresponder a un número de 138 sistemas APR, situación que dificulta la obtención de dicha información, ya que se requiere contar con el $64 \%$ de los perfiles de costos de los proyectos ejecutados en el periodo 1964-2017.

${ }^{19}$ Es una unidad de cuenta usada en Chile, reajustable de acuerdo con la inflación.

${ }^{20}$ La muestra considera valores para los ítems № de Arranque e Inversión, asociados a la puesta en marcha del proyecto y no a la evaluación económica.
} 
Tabla 1: Estadística descriptiva Población de Sistemas APR

\begin{tabular}{|c|c|c|c|c|c|c|c|c|c|c|}
\hline $\begin{array}{l}\text { Categoría } \\
\text { (o grupo) }\end{array}$ & $\begin{array}{l}\text { Número } \\
\text { de Sis- } \\
\text { temas } \\
\text { APR }\end{array}$ & Media & Medic & a Mínimo & Máximo & $\begin{array}{l}\text { Desviac } \\
\text { están- } \\
\text { dar }\end{array}$ & $\begin{array}{l}\text { Rango } \\
\text { on } \\
\text { inter- } \\
\text { cuar- } \\
\text { til }\end{array}$ & Asime & ícCurtosi\$ & $\begin{array}{l}\text { Test } \\
\text { SW: p } \\
\text { valor }\end{array}$ \\
\hline \multicolumn{11}{|c|}{ Número de Arranques } \\
\hline País & 1.806 & 233 & 153 & 14 & 3.890 & 259 & 188 & 4.47 & 40.54 & 0.000 \\
\hline $\begin{array}{l}\text { Región } \\
\text { Bio Bio }\end{array}$ & 216 & 235 & 174 & 30 & 1.421 & 199 & 171 & 2.66 & 12.27 & 0.000 \\
\hline $\begin{array}{l}\text { Periodo } \\
2010-2017\end{array}$ & 227 & 129 & 108 & 14 & 740 & 84 & 89 & 2.59 & 15.89 & 0.000 \\
\hline \multicolumn{11}{|c|}{ Beneficiarios } \\
\hline País & 1.806 & 931 & 612 & 56 & 15.560 & 1.037 & 756 & 4.47 & 40.45 & 0.000 \\
\hline $\begin{array}{l}\text { Región } \\
\text { Bio Bio }\end{array}$ & 216 & 952 & 712 & 120 & 5.684 & 799 & 699 & 2.61 & 12.04 & 0.000 \\
\hline $\begin{array}{l}\text { Periodo } \\
2010-2017\end{array}$ & 227 & 523 & 428 & 56 & 2.960 & 359 & 377 & 2.42 & 13.48 & 0.000 \\
\hline
\end{tabular}

Fuente: Elaboración propia en base a información de la DOH.

del ítem № de Arranques con un nivel de confianza del $95 \%(p<5 \%)$ y para el ítem Beneficiarios, con un nivel de confianza del $99 \%(p<1 \%)$.

Por lo tanto, el análisis comparativo sugiere que la muestra de 42 proyectos APR es representativa de la población de sistemas APR instalados a nivel de país y región del Bio Bio, por sobre la población de sistemas APR instalados en el periodo 2010-2017; estos últimos, orientados principalmente a la población semi-concentrada y dispersa, que registran un número significativamente menor de $\mathrm{N}^{\mathrm{o}}$ de Arranques y Beneficiarios.

\subsection{Evaluación económica de proyectos APR en Chile}

En el país, la tarea de realizar la evaluación económica de los proyectos APR la tiene el MDS. En las primeras etapas de evaluación, se hace uso del criterio de decisión basado en el "costo de referencia" por solución establecido para un sistema APR. ${ }^{21}$ $\mathrm{Su}$ valor se expresa en Unidades de Fomento por

\footnotetext{
${ }^{21}$ Se define como el límite de factibilidad técnico-económica de los proyectos APR. El valor vigente rige desde el año 2015 y se consideró para su determinación, una cartera de 79 proyectos de construcción de sistemas APR, lo cuales fueron ejecutados o estaban en etapa de ejecución en el periodo 20112014.
}

Arranque (UF/Arranque). Este es representando como aquel costo límite que es sugerido para la aprobación del proyecto, sin perjuicio de que si el costo de inversión por arranque del proyecto fuese mayor al costo de referencia este puede ser aprobado en instancias posteriores, conforme los cambios en las metodologías introducidas a contar del año $2014 .{ }^{22}$

Es importante destacar que en cuanto a los cambios en las metodologías de los proyectos APR, estas fueron introducidas con motivo del cumplimiento de objetivos del Programa APR para aquella población rural semi-concentrada y dispersa. A esto, se suma la adopción por parte del Estado de suministrar agua potable y saneamiento saludable, limpio, accesible y asequible para todos, conforme el reconocimiento dado al derecho humano al agua y al saneamiento, según Resolución 64/292, de fecha 28.07.2010, de la Asamblea General de las Naciones Unidas.

Ahora bien, respecto de como el MDS procede con la aplicación del criterio de decisión costo de referencia, este compara el valor referencial vigente a la fecha ${ }^{23}$ con el valor obtenido del costo de inver-

\footnotetext{
${ }^{22}$ Hasta el año 2013 se considerada como criterio el enfoque Costo Eficiencia con costo máximo, el cual indicaba que solo eran aprobados aquellos proyectos que no superaba el costo máximo. A contar del año 2014 se modifica la metodología por el enfoque de Eficiencia con costo referencial.

${ }^{23}$ Para el caso de la región del Bio Bio, el valor del costo
} 
Tabla 2: Estadística descriptiva de la muestra de sistemas APR

\begin{tabular}{|c|c|c|c|c|c|c|c|c|c|c|}
\hline Ítem & $\begin{array}{l}\text { Número } \\
\text { de Sis- } \\
\text { temas } \\
\text { APR }\end{array}$ & Media & Mediana & Mínimo & Máximo & $\begin{array}{l}\text { Desviac } \\
\text { están- } \\
\text { dar }\end{array}$ & $\begin{array}{l}\text { Rango } \\
\text { onter- } \\
\text { cuar- } \\
\text { til }\end{array}$ & Asime & aCurtosi & $\begin{array}{l}\text { Test } \\
\text { SW: p } \\
\text { valor }\end{array}$ \\
\hline $\begin{array}{l}\text { Inversión } \\
\text { en UF }\end{array}$ & 42 & 30.075 & 22.075 & 11.419 & 176.487 & 26.155 & 16.767 & 4.34 & 24.65 & 0.000 \\
\hline $\begin{array}{l}\mathrm{N}^{\mathrm{O}} \text { de } \\
\text { Arran- } \\
\text { ques }\end{array}$ & 42 & 233 & 158 & 70 & 1.448 & 260 & 125 & 3.55 & 15.71 & 0.000 \\
\hline Beneficiari & $\mathrm{s} 42$ & 1.041 & 652 & 252 & 6.323 & 1.190 & 685 & 3.39 & 14.43 & 0.000 \\
\hline
\end{tabular}

Obs. 1: La inversión se asocia al valor a ser ejecutado según presupuesto y no al monto asociado a la evaluación económica.

Obs. 2: El número de Arranques y Beneficiarios corresponde al proyecto en marcha y no al considerado en la evaluación económica.

Fuente: Elaboración propia.

sión por arranque de cada proyecto. Este último se determina en base a la inversión inicial, el cual debe estar expresado en valor neto, es decir, sin Impuesto al Valor Agregado (IVA), se restan los gastos por concepto de asesorías y gastos administrativos, ${ }^{24}$ al resultado obtenido se divide por el total de arranques del proyecto el cual debe corresponder a la situación base.

En el evento de que el proyecto APR no alcance el valor límite de aprobación, asociado al costo de referencia, el proyecto no obtiene la categoría de Recomendación Sin Condiciones (RS), es decir, no aprueba la etapa de factibilidad técnico-económica, por lo que el proyecto pasa a su reestudio donde se plantean y presentan otras alternativas semicolectivas o individuales para el suministro de agua potable. ${ }^{25}$ En esta etapa es donde la evaluación económica de los proyectos APR considera el uso de los indicadores VAC (Valor Actual de los Costos) y CAE (Costo Anual Equivalente) para seleccionar aquella alternativa de menor $\operatorname{costo}^{26}$ (incluida la original), utilizando en dichos cálculos el valor de TSD.

Por lo tanto, en el proceso de evaluación económica de los proyectos APR el énfasis esta en los requerimientos de factibilidad técnica y deja en un lugar

de referencia para el periodo 2011-2014 es igual a 130 UF y a contar del año 2015 su valor se ajusta a 144 UF.

${ }^{24} \mathrm{La}$ totalidad de los proyectos de la muestra fueron evaluados considerado el valor de la inversión inicial a valor neto (sin IVA) y excluyendo del monto de la inversión inicial el valor de las asesorías.

${ }^{25} \mathrm{La}$ totalidad de los proyectos de la muestra fueron evaluados considerado el valor de la inversión inicial a valor neto (sin IVA) y excluyendo del monto de la inversión inicial el valor de las asesorías.

${ }^{26}$ Estos indicadores se basan en el enfoque Costo Eficiencia. secundario la evaluación económica de alternativas que se puedan plantear (inicialmente) para dar solución al problema de suministro de agua potable. Esta situación se presenta como observación al sistema de evaluación, toda vez pierden importancia aspectos económicos de los proyectos, por sobre requerimientos de tipo técnico.

Lo anterior, se verifica para el caso de los 42 proyectos APR de la región del Bio Bio, donde se presenta un bajo nivel de oferta de alternativas de solución (colectiva) destinada al problema del suministro de agua potable rural. Esta situación, en parte tendría su origen, en los bajos niveles de innovación en los procesos de diseño de los sistemas APR, los que se han mantenido sin cambios significativos desde los años sesenta. Sobre este aspecto, es importante destacar que en el país, la cantidad de alternativas de solución factibles a desarrollar es reducida y corresponde a un conjunto de 6 (seis) tipos de abastecimiento de agua potable, ${ }^{27}$ conforme se indican en "'Estudio de Saneamiento Rural" efectuado por la empresa Homsi y Asociados Ltda (2007), encargado por la Subsecretaria de Desarrollo del Ministerio del Interior, Chile.

Además, respecto de las 6 (seis) alternativas de sistemas APR que se han definido como factibles de ser instalados en el país, se observa que estas presentan problemas importantes, según consta en el informe realizado por la DOH al Programa de Infra-

\footnotetext{
${ }^{27}$ En la región del Bio Bio se presentan principalmente dos tipos de abastecimiento de agua potable, aquellos basados desde fuentes de aguas subterráneas con estanque enterrado, que corresponden al $28.57 \%$ de la muestra y los sistemas basados desde fuentes de agua subterránea con estanque elevado, que corresponden al $64.29 \%$ de la muestra.
} 
Tabla 3: Test de diferencia de medias

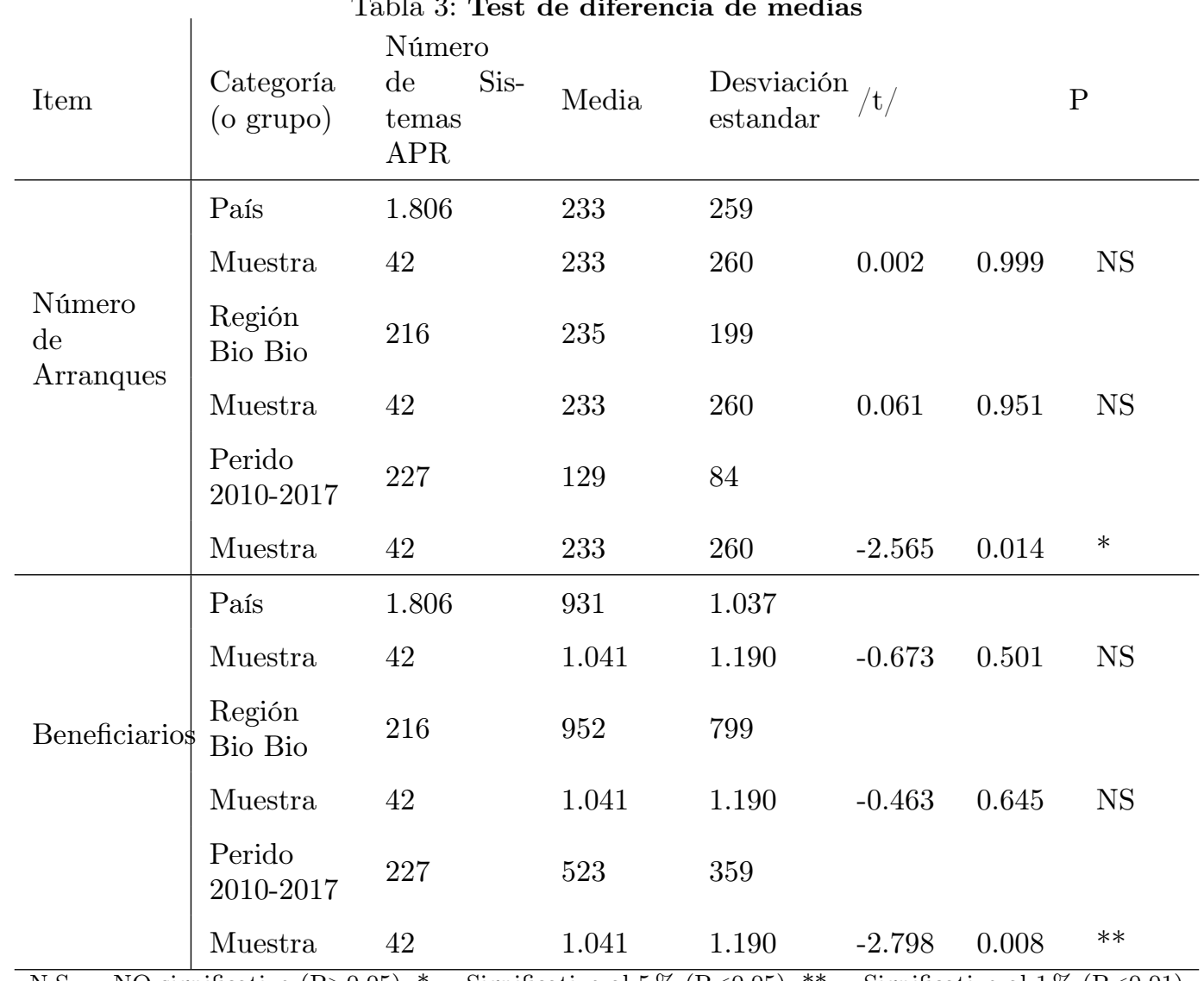

Hipótesis Nula: Igualdad de Medias para el Item respectivo (№ Arranques, Beneficiarios), entre el grupo de Sistemas APR instalados a nivel de cada categoría (País, Región Bio Bio y Periodo 2010-2017) versus Muestra de proyectos APR.

Fuente: Elaboración propia.

estructura Hidráulica de APR (2011-2014), donde sistemas APR que cuentan incluso con menos de 20 años, un porcentaje importante presenta fallas en su funcionamiento. ${ }^{28}$

\section{Metodología}

\subsection{Funciones de descuento}

Para realizar el análisis económico de los 42 proyectos APR se consideran 3 (tres) tipos de factor de descuento. El primero está basado en la función de descuento exponencial, tradicionalmente utilizado por la teoría económica para realizar la evaluación de los proyectos del sector público; los dos restantes, están basados en los enfoques de tasas de descuentos decrecientes, correspondientes a la fun-

\footnotetext{
${ }^{28}$ En los años 2013 y 2014 se presentaron cortes no programados ocasionados por fallas de infraestructura de los sistemas APR, correspondientes al $20.29 \%$ y $23.74 \%$ de los sistemas APR existentes en los años 2013 y 2014.
}

ción de descuento hiperbólica de un parámetro ${ }^{29}$ y la función hiperbólica generalizada, esta última representada por los estudios realizados por Poulos y Whittington (2000), el cual aplica el factor hiperbólico a vidas humanas. ${ }^{30}$

En la Figura 2 se representa el valor actual de una unidad monetaria medida en pesos considerando los tres tipos de funciones de descuento, calculado para un valor de TSD igual a $6 \%$ (valor vigente año 2018) y un periodo de 50 años.

En el gráfico se observa que el valor actual de una unidad monetaria en el año 50 es mayor para el factor hiperbólico de un parámetro (color rojo), que da mayor peso a flujos de beneficios netos en pe-

\footnotetext{
${ }^{29} \mathrm{La}$ formula de la función de descuento se indica en la Sección 2.2 de este documento.

${ }^{30} \mathrm{El}$ factor hiperbólico generalizado se define como $F G_{g}=$ $\frac{1}{(1+a t)^{a / b}}$, donde $a, b>0$; se considera en este estudio que el valor de los parámetros se definan como $a=b=2 r$, donde $r$ es la tasa de descuento a aplicar.
} 
Figura 2: Funciones de descuento

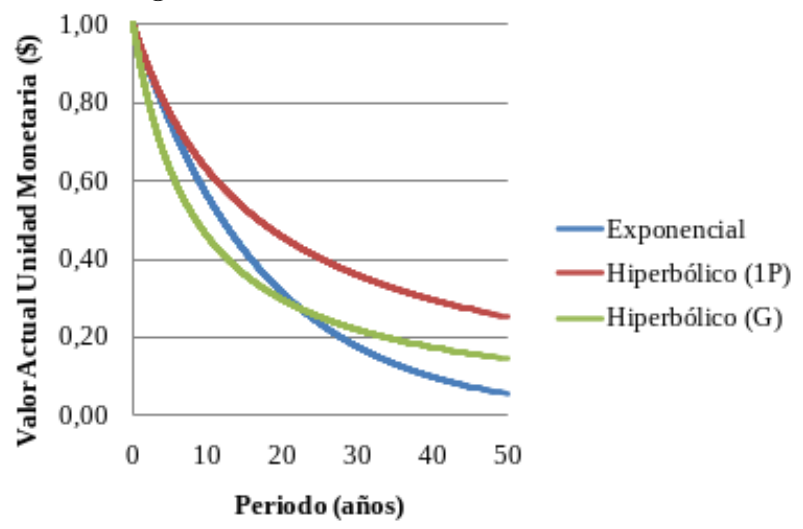

Fuente: Elaboración propia.

riodos lejanos. El valor actual intermedio se obtiene con el factor hiperbólico generalizado (color verde), que descuenta los flujos de beneficios netos a tasas más rápidas en periodos cercanos, por sobre periodos lejanos del proyecto y finalmente el menor valor actual es obtenido es con el uso del factor exponencial (color azul), que da mayor peso a los flujos de beneficios netos en periodos cercanos.

Para un horizonte de 20 años que es lo que representa la vida útil de un proyecto APR, el factor hiperbólico generalizado aplica un mayor descuento al flujo de una unidad monetaria en los periodos que dura el proyecto, igualando aproximadamente su valor al factor exponencial para periodos cercanos al año 20. Por su parte, el factor hiperbólico de un parámetro es el que menor descuento aplica a los flujos.

Lo anterior, se verifica tanto para proyectos que registren flujos de costos de valor constante, como así también variable para cada periodo que dura el proyecto. Esta última situación queda representada para el caso de proyectos donde los flujos de costos pueden ser creciente en el tiempo (debido a mayores costos en electricidad, productos químicos y mantención) u oscilar de un periodo a otro, producto de reinversiones realizadas en periodos intermedios (inversión en mejoramiento y/o reparación), tal como se indica en la Figura 3 para el caso del proyecto APR No 21 ' 'Las Vegas" perteneciente a la Provincia del Bio Bio, donde en el periodo 10, se registran niveles de costos significativamente distintos a los otros periodos, producto de reinversiones realizadas al sistema APR.
Figura 3: Función de descuento para flujos de los costos

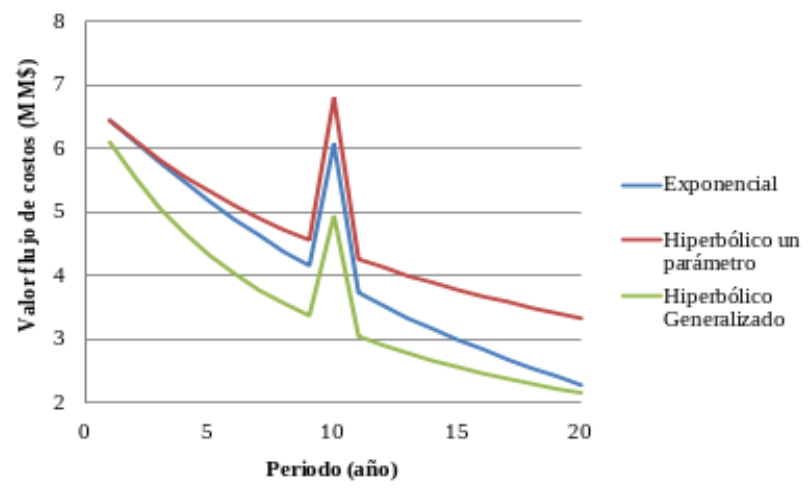

Fuente: Elaboración propia.

\subsection{Tasa de Descuento Límite de Aproba- ción de Proyectos APR}

Si bien, la evaluación económica de los proyectos considera un enfoque Eficiencia, es decir, no considera beneficios cuantificables que los proyectos puedan aportar a la comunidad rural y en consideración, a que en el proceso de evaluación de proyectos APR se da énfasis al enfoque de eficacia técnica de los proyectos (representada por el costo de referencia), por sobre el enfoque de eficiencia económica (evaluar distintas alternativas), se plantea como supuesto para esta investigación que el beneficio mínimo cuantificable obtenido de los proyectos APR sea representado por el valor del costo de referencia.

En consideración a lo anterior, se buscará obtener un valor de tasa de descuento que sea proxy a la tasa costo de oportunidad del proyecto (tasa $\operatorname{TIR}^{31}$ ), el cual será definido como Tasa de Descuento Límite de Aprobación (TDLA) del proyecto. Para estimar esta tasa se requieren obtener el VAC por arranque de cada proyecto, considerando los flujos de los costos para su vida útil (20 años), conforme se indican en cada perfil de proyecto. Luego, este valor se iguala con el valor asociado al costo de referencia. La tasa de descuento que permite igualar ambos valores y dar por aprobado el proyecto, corresponderá a la TDLA que será proxy de la tasa costo de oportunidad del proyecto APR.

Para adoptar dicho procedimiento, se consideran algunas condiciones al proceso de evaluación eco-

\footnotetext{
${ }^{31}$ Conforme el enfoque de evaluación económica Costo Beneficio, la TIR representa la Tasa Interna de Retorno, que permite obtener un Valor Actual de los Beneficios Netos del proyecto igual a cero $(V A N=0)$.
} 
nómica de los proyectos APR, los que se indican a continuación:

- Reflejar los valores de la inversión inicial y los flujos de costos de cada periodo a precios sociales, esto implica eliminar distorsiones de mercado tales como los impuestos (IVA) y aplicar el Factor de Corrección Social (FCS) respectivo.

- No considerar el gasto asociado a las asesorías técnicas en el monto de la inversión inicial de cada proyecto.

- Aplicar FCS respectivo al costo de referencia.

En relación a la información asociada a los perfiles de costos de los 42 proyectos APR, es importante destacar que los proyectos $\mathrm{N}^{\mathrm{O}} 2,4,6,15,16,18$, $20,22,24,25,33,34,36,39,40$ y 42 se les aplico al monto de la inversión inicial (sin IVA), un FCS igual a 0.9 por ser este valor representativo en el ajuste realizado al ítem inversión inicial de los proyectos APR; toda vez en los perfiles de los proyectos no indicaban el valor del FCS a ser aplicado en la evaluación económica.

Además, en los perfiles de costos de los proyectos № $8,11,13$ y 23 solo contaban con información registrada a precios privados (no sociales). Por lo que se procedió a deducir del valor de la inversión inicial los gastos de las asesorías y expresar el valor de la inversión inicial, como así también el valor de los flujos de costos, a valores netos (sin IVA), al resultado obtenido se multiplicó por 0.9 correspondiente FCS promedio.

\subsection{Función Tasa de Aprobación de Proyec- tos}

Como fue mencionado en el proceso de obtención de la TDLA se recurrió al uso del indicador económico VAC, estimado por arranque para cada uno de los 42 proyectos. Este indicador, tal como lo sugiere la teoría económica, muestra una relación aproximadamente lineal negativa entre las variables VAC y TSD. En la Figura 4 se muestra la gráfica estimada para 17 puntos del VAC por arranque del proyecto № 2 ' El Zarzal - Monteblanco - Calle Dávila" de la Comuna El Carmen, Provincia de Nuble.

En el gráfico se observa una línea vertical en color verde, perpendicular al eje de las abscisas y fijada en el valor de TSD igual a $10.069 \%$, que corresponde al valor de la TDLA. Esta línea indican dos posi-
Figura 4: VAC por arranque vs Tasa Social de Descuento

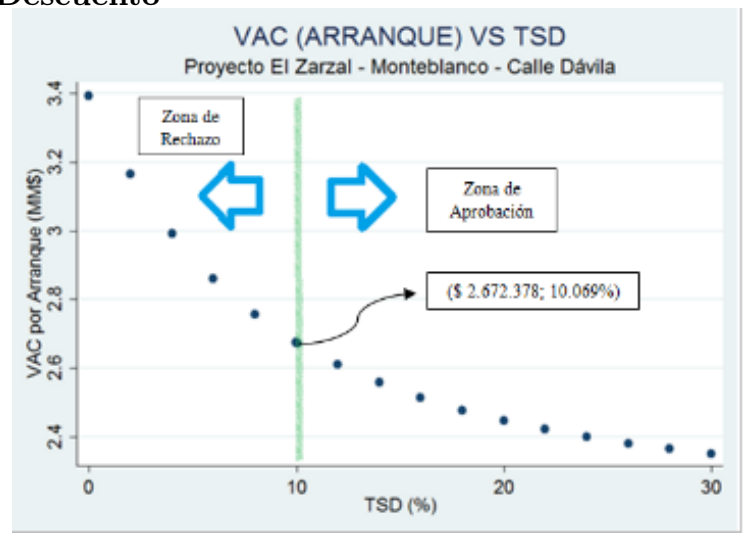

Fuente: Elaboración propia..

bles resultados obtenidos de la evaluación económica del proyecto, un valor de TSD igual o superior al $10.069 \%$ el proyecto se ubica en la Zona de Aprobación, ya que el VAC por arranque será igual o menor al valor costo de referencia, que corresponde a un monto de $\$ 2.672 .368$. Por su parte, para un valor de TSD inferior al $10.069 \%$ y cercano cero, el proyecto se ubica en la Zona de Rechazo, ya que el VAC por arranque del proyecto será mayor al valor del costo de referencia.

Lo anterior es importante a ser destacado, ya que al evaluar individualmente un proyecto APR, con aplicación del criterio de decisión basado en TDLA, el proyecto registra más de un valor para la TSD que permite ser aprobado, como así también registra más de un valor de TSD que permiten ser rechazado.

Respecto de la función Tasa de Aprobación de Proyectos (TAP), esta es definida para una cartera de proyectos APR. Si bien su valor está entre 0 y 1 ; esta se expresa en valores porcentuales (\%) e indica la proporción de proyectos APR que son aprobados para un determinado valor de TSD. En el Anexo 2 se indican un rango de valores para la función TAP que van del 28.6 al $100 \%$, definidos para cada nivel de TSD. Por su parte, la Figura 5 muestra la representación gráfica de la función TAP con uso del factor de descuento exponencial, ${ }^{32}$ definido para un rango de TSD del 0 al $52 \%$.

En la gráfica es posible observar que para un ran-

\footnotetext{
${ }^{32}$ Para definir los valores de necesarios para representar gráficamente la función TAP, estos son obtenidos de fijar en el valor de $T S D_{0}=0 \%$, y considerar a partir de este punto, sucesiones aritméticas de números en sentido creciente, con una diferencia igual al $1 \%$.
} 
Figura 5: Función TAP para valor de TSD de 0 al $52 \%$

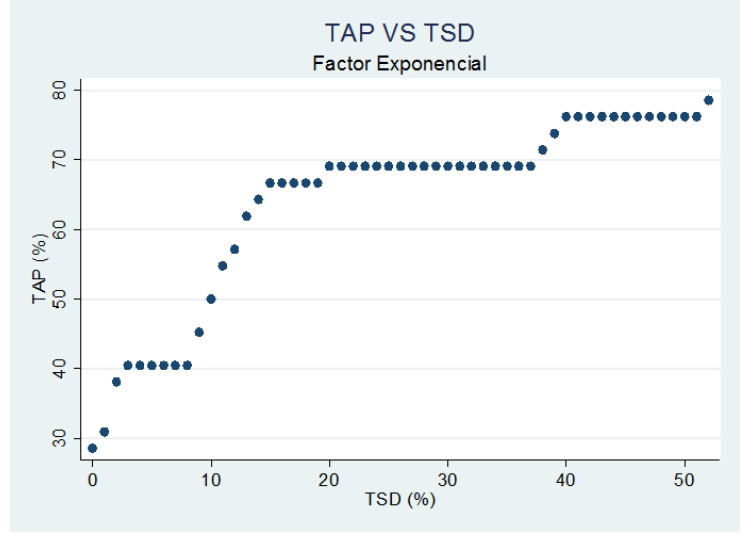

Fuente: Elaboración propia.

go de TSD que va del 3 al $8 \%$, intervalo donde se encuentra el valor de la TSD igual al $6 \%$ (valor vigente año 2018), se registra una TAP del 40.5\%, que indican que 17 de los 42 proyectos APR son aprobados. Además, se observa que la curva TAP presenta saltos, lo que implica que para determinados rangos de TSD, corresponden solo un valor de TAP. La presencia de valores constantes para la variable TAP, tal como se indico anteriormente, se explica por el hecho de que cada proyecto APR en forma individual puede presentar dos posibles resultados. ${ }^{33}$

Además, la gráfica muestra una relación positiva (creciente) entre la TAP y la TSD, esto debido a que la cartera de proyectos a la cual se realiza la evaluación económica, constituida por 42 proyectos APR, los valores de la TDLA al ser distintos para cada proyecto, requieren diferentes valores de TSD que permitan igualar con la TDLA y dar por aprobado el proyecto. ${ }^{34}$

Por su parte, en la Figura 6 se muestran las gráficas de las funciones TAP con uso del factor de descuento hiperbólico de un parámetro e hiperbólico generalizado para un rango de valores de TSD del 0 al $50 \%$. Estas gráficas son obtenidas en base al mismo procedimiento adoptado para el caso del factor exponencial. Se observa que el comportamiento de ambas curvas presenta similitudes en cuanto a la forma de la curva obtenida para el caso del factor exponencial, como así también valores constantes y

\footnotetext{
${ }^{33} \mathrm{El}$ proyecto es aprobado para un valor de TSD mayor o igual a la TDLA o es rechazado cuando el valor de la TSD es menor a la TDLA.

${ }^{34} \mathrm{El}$ proyecto es aprobado para un valor de TSD mayor o igual a la TDLA o es rechazado cuando el valor de la TSD es menor a la TDLA.
}

tendencia creciente de la variable TAP.

Además, se observa que para un valor de TSD igual al $6 \%$, ambos factores de descuento, registran una TAP igual a 40.5\%, es decir, son aprobados 17 de los 42 proyectos APR, tal como en el caso del factor exponencial. Sin embargo, estos factores de descuentos registran distintos rangos de valores de TSD que permiten alcanzar ese mismo nivel de aprobación, donde el factor hiperbólico de un parámetro corresponde del 3 al $12 \%$ y el factor hiperbólico generalizado del 2 al $6 \%$. Esta diferencia es significativa, ya que frente a un alza en el valor de la TSD, por sobre el valor del $6 \%$, con uso de un factor de descuento hiperbólico generalizado se incrementa la cantidad de proyectos aprobados.

En la Tabla 4 se indican los estadísticos asociados a la correlación presente entre las variables. Se observa que la relación no es de tipo lineal, aunque sí aproximadamente monótona creciente, toda vez los valores asociados a la correlación de Pearson son menores a los de Spearman. Además, se rechaza con un nivel de confianza del $99 \%(\mathrm{p}<0.01)$ la hipótesis nula de independencia estadística, lo que sugiere una asociación significativa entre ambas variables. 
Figura 6: Función TAP de tasas de descuento decrecientes
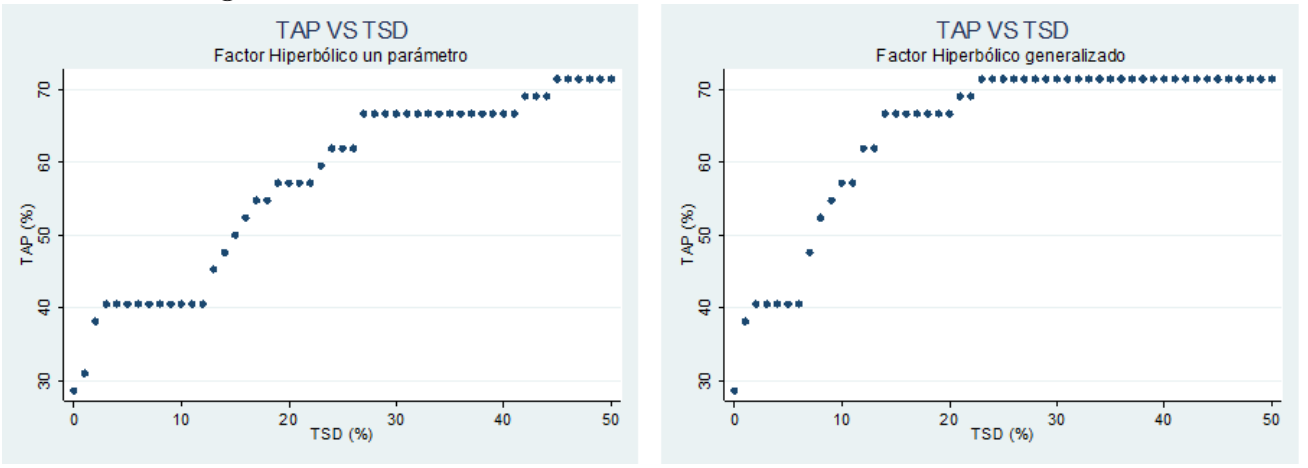

Fuente: Elaboración propia..

Tabla 4: Correlación TAP y TSD

\begin{tabular}{|c|c|c|c|c|c|}
\hline \multirow{2}{*}{$\begin{array}{l}\text { Factor de descuento } \\
\text { Exponencial }\end{array}$} & \multirow{2}{*}{$\begin{array}{l}\text { Estadístico } \\
\text { Pearson }\end{array}$} & \multirow{2}{*}{$\frac{\mathrm{N}^{\mathrm{o}} \text { de Proyectos }}{53}$} & \multirow{2}{*}{$\begin{array}{c}\text { valor } \\
0.879\end{array}$} & \multicolumn{2}{|c|}{ Student } \\
\hline & & & & 0.000 & $* *$ \\
\hline & Spearman & & 0.973 & & \\
\hline \multirow[t]{2}{*}{ Hiperbólico un parámetro } & Pearson & 51 & 0.949 & 0.000 & $* *$ \\
\hline & Spearman & & 0.982 & & \\
\hline \multirow[t]{2}{*}{ Hiperbólico generalizado } & Pearson & 51 & 0.809 & 0.000 & $* *$ \\
\hline & Spearman & & 0.912 & & \\
\hline
\end{tabular}

Fuente: Elaboración propia.

\subsection{Elasticidad función TAP}

La representación gráfica obtenida para la función TAP indica que esta se asocia a una función de distribución discreta monótonamente creciente, donde la elasticidad de la TAP con respecto a la TSD no es constante.

Para estimar la elasticidad de la función TAP, se considera en los cálculos el método de la elasticidad arco, que si bien no es preciso en la determinación del valor de la elasticidad según lo propuesto por el enfoque analítico, ${ }^{35}$ proporciona estimaciones aproximadas. La fórmula para la elasticidad arco viene dada por:

$$
\begin{array}{r}
E_{T A P ; T A P}=\frac{\delta \% T A P}{\delta \% T S D}= \\
{\left[\frac{T A P_{j}-T A P_{i}}{T S D_{j}-T S D_{i}}\right] *\left[\frac{T S D_{i}+T S D_{j}}{T A P_{i}+T A P_{j}}\right]}
\end{array}
$$

\footnotetext{
${ }^{35}$ Para una función continua $Y=f(X)$, viene dada por la formula: $E_{Y / X}=\frac{\left[\frac{d y}{y}\right]}{\left[\frac{d x}{x}\right]}=\frac{\frac{d y}{d x} * x}{y} ;$ donde $\frac{d y}{d x}$ corresponde a la pendiente de la función.
}

Donde,

$\left.T S D_{j}-T S D_{i}\right)=2 \%$, se obtiene de la diferencia de $T S D_{j}=\left(T S D_{n}+1 \%\right)$ y $T S D_{i}=\left(T S D_{n}-1 \%\right)$. Valores que permiten estimar $T A P_{j}$ y $T A P_{i}$. Con $i$, $j$ y $n \in$ a los números enteros.

Los valores de elasticidad estimados para la función TAP, son útiles como herramienta para la $\mathrm{Au}-$ toridad económica, ya que le permiten predecir lo que es probable que ocurra con la cantidad aprobada de proyectos, cuando existen cambios en el valor de la TSD. Estos resultados se obtienen cuando se reordena la formula de la elasticidad de la TAP, de la siguiente forma:

$$
\frac{\delta T A P}{T A P_{i}}=\left[E_{T A P ; T A P}\right] *\left[\frac{\delta T S D}{T S D_{i}}\right]
$$




\section{Resultados}

\subsection{Análisis de la TDLA}

En la Anexo 3 se indican los valores de la TDLA para cada uno de los 42 proyectos APR, los que son estimados para cada factor de descuento (exponencial e hiperbólico). Se observa que, para un criterio de decisión donde se aprueban aquellos proyectos que registren un valor de TDLA menor o igual a un valor de TSD igual al 6\%, se aprueban 17 de los 42 proyectos $(\mathrm{TAP}=40.5 \%$ ). Este resultado es igual para cada uno de los tres factores de descuento utilizado en la evaluación económica. Por su parte, con aplicación del criterio de decisión basado en el costo de referencia que aplica el MDS, se aprueban 34 de los 42 proyectos ( $81 \%$ de la muestra). ${ }^{36}$

En la Tabla 5 se presenta cuadro resumen con los valores de la correlación de Pearson y Spearman, para la variable TDLA con respecto a cada ítem del proyecto (Inversión, № de Arranques y Beneficia$\operatorname{rios}^{37}$ ), aplicada para una muestra de 34 y 32 proyectos $\mathrm{APR}^{38}$ y estimados para cada uno de los factores de descuento. Los resultados sugieren que no existe relación de tipo lineal y/o algún otro tipo de dependencia funcional significativa entre la TDLA y la inversión. Es decir, valores de TDLA elevados lo podemos encontrar tanto en proyectos con montos de inversión elevados o bajos (y viceversa).

Por su parte, la TDLA respecto de los ítems del proyecto No de Arranques y Beneficiarios, presenta un nivel de relación lineal bajo y negativo. Sin perjuicio de lo anterior, con un nivel de confianza del $99 \%(\mathrm{p}<0.01)$ y $95 \%(\mathrm{p}<0.05)$, se rechazan la hipótesis nula que indica que la variable TDLA con respecto a los ítems del proyecto $\mathrm{N}^{\mathrm{O}}$ de Arranques y Beneficiarios respectivamente, son independientes. Esto indica una relación funcional significativa entre dichas variables, lo que sugiere que proyectos que presenten bajo $\mathrm{N}^{\mathrm{o}}$ de Arranques y Beneficiarios, pueden presentar una TDLA de valor alto en comparación al promedio.

\footnotetext{
${ }^{36} \mathrm{El}$ criterio consiste en que el costo inversión por arranque del proyecto debe ser menor o igual al costo de referencia.

${ }^{37}$ Los valores asociados a los ítems Inversión y № de Arranques, corresponde a valores utilizados en la evaluación económica y el ítem Beneficiarios, a la puesta en marcha del proyecto.

${ }^{38}$ Para la muestra $\mathrm{n}=34$ se excluyen de la muestra inicial $(\mathrm{n}=42)$ los proyectos № $4,6,10,15,16,19,34$ y 37; toda vez la TDLA registra un valor elevado (con tendencia al infinito). Por su parte, para la muestra $\mathrm{n}=32$, son excluidos de la muestra $\mathrm{n}=34$, los proyectos $\mathrm{N}^{\mathrm{o}} 1$ y 29 , que se destinan a una población superior a la concentrada (más de 3.000 habitantes).
}

\subsection{Análisis de la elasticidad de la función TAP}

La elasticidad para la función TAP estimada para cada factor de descuento se indican en la Tabla 6. Esta considera un rango de valores de TSD del 0 al $20 \%$. En general se observa que los niveles de elasticidad son bajos para cada factor de descuento (inelástica $^{39}$ ) y en algunos rangos de TSD la elasticidad es igual a cero (nula). Este último resultado se observa para el rango de TSD del 4 al $7 \%$ para el factor exponencial, del 4 al $11 \%$ para el factor hiperbólico de un parámetro y del 3 al $5 \%$ para el factor hiperbólico generalizado.

Para un valor del TSD igual al $6 \%$, el factor exponencial e hiperbólico de un parámetro presentan elasticidad igual a cero (nula), lo que implica que variaciones del orden del $16.6 \%$ al alza o a la baja en el valor de la TSD, es decir, se incrementa su valor del 6 al $7 \%$ o disminuye su valor del 6 al $5 \%$, no provocan cambios en la TAP. Esto se traduce en que se mantiene el nivel de TAP igual al 40.5\% (se aprueban 17 de los 42 proyectos).

Por su parte, para un valor de TSD igual al $6 \%$ el factor hiperbólico generalizado presenta una elasticidad mayor a cero. Este resultado sugiere que para el promedio de TSD que va desde el 5 al $7 \%$, se debe registrar una elasticidad promedio de 0.49 en la TAP. ${ }^{40}$ Esta elasticidad se aplica tanto para un aumento en el valor de la TSD (del 5 al $6 \%$ ), como en una disminución del valor de la TSD (del 7 al $6 \%$ ). Sin embargo, un desplazamiento del valor de la TSD que va desde el 6 al $5 \%$, no se registra elasticidad en la TAP, toda vez la elasticidad promedio para un valor de TSD igual al $5 \%$ es cero (nula).

Por lo tanto, los resultados obtenidos para la elasticidad de la función TAP, analizados en un contexto de políticas públicas, que incentive la instalación de sistemas APR en la región del Bio Bio por parte del Estado, sugieren que con independencia del uso del factor de descuento aplicados en los procesos de evaluación económica de los proyectos, en un escenario de disminución en el valor de la TSD, no se verán afectados los niveles de TAP.

\footnotetext{
${ }^{39} \mathrm{El}$ valor de la elasticidad en términos absolutos está entre 0 y 1 .

${ }^{40}$ Un desplazamiento del valor de la TSD del 5 al $6 \%$ (que implica aumento del $20 \%$ ), provocan un desplazamiento en la TAP del 40.5 al $44.5 \%$ (aumenta en un $9.8 \%$ ). Por su parte, un desplazamiento del valor de la TSD del 7 al $6 \%$ (que implica una disminución del $14.3 \%$ ), provocan un desplazamiento en la TAP del 47.6 al $44.3 \%$ (disminuye un $7 \%$ ).
} 


\section{Tabla 5: Correlación TDLA versus Ítem del proyecto APR}

$$
\mathrm{N}^{\mathrm{O}} \text { de }
$$

Item del Estadístico Pro-

Proyecto yectos

Exponencial Hiperbólico de un Parámetro Hiperbólico Generalizado

\begin{tabular}{|c|c|c|c|c|c|c|c|c|c|c|c|}
\hline & & & $\begin{array}{l}\text { valor } \\
\mathrm{t}\end{array}$ & Stud & nt $P$ & $\begin{array}{l}\text { valor } \\
\mathrm{t}\end{array}$ & & & $\begin{array}{l}\text { valor } \\
\mathrm{t}\end{array}$ & & $\mathrm{P}$ \\
\hline \multirow[t]{2}{*}{ Inversión } & Pearson & 34 & 0.081 & & & 0.096 & & & 0.096 & & \\
\hline & Spearman & & 0.021 & 0.907 & NS & 0.023 & 0.899 & NS & 0.026 & 0.883 & NS \\
\hline \multirow{2}{*}{$\begin{array}{l}\mathrm{N}^{\mathrm{O}} \text { de } \\
\text { Arranques }\end{array}$} & Pearson & 34 & $\begin{array}{l}- \\
0.203\end{array}$ & & & $-\overline{0.157}$ & & & $\begin{array}{l}- \\
0.158\end{array}$ & & \\
\hline & Spearman & & $\begin{array}{l}- \\
0.467\end{array}$ & 0.005 & $* *$ & $-\overline{0.454}$ & 0.007 & $* *$ & $\begin{array}{l}- \\
0.447\end{array}$ & 0.008 & $* *$ \\
\hline \multirow[t]{2}{*}{ Beneficiarios } & Pearson & 34 & $-\overline{0.172}$ & & & - & & & $\begin{array}{l}- \\
0.138\end{array}$ & & \\
\hline & Spearman & & $\begin{array}{l}- \\
0.365\end{array}$ & 0.034 & $*$ & $-\overline{0}$ & 0.041 & $*$ & $\begin{array}{l}- \\
0.346\end{array}$ & 0.046 & $*$ \\
\hline \multirow[t]{2}{*}{ Inversión } & Pearson & 32 & $\begin{array}{l}- \\
0.169\end{array}$ & & & $\begin{array}{l}- \\
0.168\end{array}$ & & & $\begin{array}{l}- \\
0.168\end{array}$ & & \\
\hline & Spearman & & $\begin{array}{l}- \\
0.002\end{array}$ & 0.992 & NS & $-\overline{0}$ & 0.997 & NS & 0.002 & 0.994 & NS \\
\hline \multirow[t]{2}{*}{$\begin{array}{l}\mathrm{N}^{\mathrm{O}} \text { de } \\
\text { Arranques }\end{array}$} & Pearson & 32 & 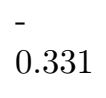 & & & $-\overline{0}-269$ & & & $\begin{array}{l}- \\
0.269\end{array}$ & & \\
\hline & Spearman & & $\begin{array}{l}- \\
0.491\end{array}$ & 0.004 & $* *$ & $-\overline{0.476}$ & 0.006 & $* *$ & $\begin{array}{l}- \\
0.472\end{array}$ & 0.006 & $* *$ \\
\hline \multirow[t]{2}{*}{ Beneficiarios } & Pearson & 32 & - & & & 0.214 & & & $\begin{array}{l}- \\
0.214\end{array}$ & & \\
\hline & Spearman & & $\begin{array}{l}- \\
0.369\end{array}$ & 0.038 & $*$ & $-\overline{0.355}$ & 0.047 & $*$ & $\begin{array}{l}- \\
0.349\end{array}$ & 0.049 & $*$ \\
\hline
\end{tabular}

N.S. $=$ NO significativo $(\mathrm{P}>0.05){ }^{*}=$ Significativo al $5 \%(\mathrm{P}<0.05) ; * *=$ Significativo al $1 \%(\mathrm{P}<0.01)$

Fuente: Elaboración propia.

Rev. Est. de Políticas Públicas, 5(2): julio 2019 - noviembre 2019 


\subsection{Pruebas de estabilidad de los datos pa- ra los proyectos APR}

Con el objetivo de analizar la robustez y consistencia de los valores obtenidos para la TDLA y la elasticidad de la función TAP, se realizan pruebas de estabilidad de los datos para el conjunto de 42 proyectos APR. Estas pruebas consisten en comparar los resultados de la evaluación económica de los proyectos APR de la región del Bio Bio versus los resultados obtenidos para los mismos proyectos, pero instalados en otra zona del país.

Para llevar a cabo estas pruebas, en primer lugar se define el diferencial de los costos de referencia para la construcción de los sistemas APR de las distintas zonas del país ${ }^{41}$ con respecto a la zona 3 (donde se encuentra inserta la región del Bio Bio). Este diferencial en costos puede ser explicado por diversos factores, entre ellos; diferencias en costos de solución de los sistemas APR, disponibilidad de terrenos para la instalación de los sistemas APR, derechos de agua adquiridos, entre otros.

Luego, se procede a incorporar el valor obtenido correspondiente a la diferencia del costo de referencia de cada zona en los perfiles de costos de los 42 proyectos APR, determinando con ello el valor de la TDLA ${ }^{42}$ y la elasticidad de la función TAP para cada una de las 6 zonas del país, haciendo presente que en los cálculos realizados, se considero solo el uso del factor de descuento exponencial.

En la Tabla 7, se indica el valor asociado al costo de referencia para la construcción de sistemas $\mathrm{APR}^{43}$ (columna A), el cual es determinado por el MDS en base al costo de inversión promedio por arranque de cada zona ${ }^{44}$ (columna C). Además, en dicha Tabla 7s posible observar el resultado obtenido correspondiente a la diferencia en costos de referencia de las distintas zonas del país con respecto a la

\footnotetext{
${ }^{41}$ En Chile el MDS considera la existencia de 6 zonas. Cada zona puede contar con más de una región del país. Para el caso de la zona 3, esta considera las regiones Metropolitana, Libertador Bernardo O Higgins, Maule y del Bio Bio.

${ }^{42} \mathrm{El}$ valor de la TDLA corresponde a la tasa de descuento del proyecto necesaria para igualar el valor del costo de referencia fijado por el MDS con el VAC por arranque del proyecto.

${ }^{43}$ Como se menciono en la Sección 2.4, su valor está vigente desde el año 2015.

${ }^{44} \mathrm{El}$ procedimiento de ajuste del valor del costo de referencia para cada zona, se realiza en base a un conjunto de proyectos de sistemas APR, donde determina el costo promedio por arranque para cada una de las 6 zonas, al que aplica un factor de 1.3 (promedio ajustado), al resultado obtenido se compara con los costos máximos vigentes de cada zona y si este es mayor, se ajusta su valor al alza.
}

zona 3 (columna E).

Los resultados obtenidos se indican en la Tabla 8, donde se observa que al aplicar el criterio de decisión basado en aprobar aquellos proyectos APR que registren un valor de TDLA menor o igual al $6 \%$, en las otras 5 zonas del país se registran diferencias significativas en la cantidad de proyectos aprobados respecto de los registrados en la zona 3 , donde se aprueban 17 de los 42 proyectos.

En cuanto a los valores de elasticidad de la función TAP estimadas para un rango de valores de TSD del 0 al $20 \%$. En ella es posible observar que para valores de TSD del 4 al $7 \%$, las otras 5 zonas del país presentan niveles de elasticidad bajos (inelástica) en comparación con los valores obtenidos para la zona 3 , que registra elasticidad igual a cero (nula).

Por lo tanto, los resultados obtenidos permiten concluir la presencia de diferencias significativas en los valores para la TAP y elasticidad de la función TAP de las otras 5 zonas del país con respecto a la zona 3. Además, tomando en consideración que el MDS para efectos de determinar el costo de referencia vigente para cada zona del país, utiliza una base reducida de proyectos $\mathrm{APR},{ }^{45}$ se estimó un nuevo costo promedio por arranque para la zona 3 , considerando en los cálculos la muestra de 42 proyectos APR.

Para ello, se dividió la muestra en dos grupos, proyectos aprobados en el periodo 2012-2014, que registraron un costo promedio por arranque de $108.7 \mathrm{UF}$ y proyectos aprobados en el periodo 2015-2017, que registraron un costo promedio por arranque de 116.8 UF. ${ }^{46}$ Con aplicación del factor 1.3 se obtienen como valor de costo referencia los montos de 141 y 152 UF respectivamente. Con los valores obtenidos para los nuevos montos de costo de referencia, se determinan los nuevos valores de TDLA y elasticidad de la función TAP para la zona 3 .

En la Tabla 9 se indican los resultados, donde es posible observar que para un nivel de TSD igual al $6 \%$, la cantidad aprobada de proyectos de la zona 3 resulta ser similar para el caso de proyectos aprobados en las zona 4 y 6 , las que registran costos de refe-

\footnotetext{
${ }^{45}$ Para su cálculo se considero una cartera constituida por 79 proyectos de construcción de sistemas APR en el periodo 2011-2014.

${ }^{46} \mathrm{El}$ grupo de proyectos aprobados periodo 2012-2014 considera los proyectos $2,3,6,8,13,15,16,18,19,20,23,24$, $33,34,35,39,40$ y 42 . Para el grupo de proyectos aprobados periodo 2015-2017 considera los proyectos $1,4,5,7,9,10,11$, $12,14,17,21,22,25,26,27,28,29,30,31,32,36,37,38,41$.
} 
rencia de 175 y 163 UF respectivamente. En cuanto a la elasticidad de la función TAP, si bien para un valor de TSD igual al $6 \%$ los valores de elasticidad de la zona 3 son superiores a las zonas 4 y 6 , para el rango de valores de TSD del 4 al $7 \%$, todas las zonas del país registran niveles de elasticidad en la TAP en promedio similares (inelástica).

Por lo tanto, esta última prueba permitió observar que aplicando ajustes al alza en el valor del costo de referencia de la zona 3, los resultados de la TAP y la elasticidad de la función TAP, para la cartera de proyectos APR de la región del Bio Bio, son consistentes con los resultados obtenidos al evaluar esos mismos proyectos en otras zonas del país. Lo cual sugiere, que el valor del costo de referencia que ha definido el MDS para la zona 3, no está bien ajustado.

Un punto a destacar respecto de la zona 3, que registra un valor del costo de referencia de $144 \mathrm{UF}$ (vigente año 2018). Es que esta exigencia también se aplica a las regiones Metropolitana, O Higgins y del Maule; hecho que puede ser cuestionable, toda vez no consideran otros aspectos que son propios de cada región, como por ejemplo la realidad hídrica, donde la disponibilidad del recurso agua difiere significativamente entre estas regiones.

Lo anterior, lo hace presente la Dirección General de Aguas (DGA) el año 2016, donde señala que escases del recurso se presenta principalmente en las Macrozonas del Norte y Centro del país, tal como se observa en la Figura 7, las que están bajo el promedio internacional ${ }^{47}$ en cuanto al nivel de escorrentía media anual. ${ }^{48}$

Respecto de la región del Bio Bio, que pertenece a la Macrozona Sur, para el año 2016 registraba un valor por escorrentía per cápita de 24.432 $\mathrm{m} 3$ /persona/año, superior al obtenido en las regiones Metropolitana, O Higgins y del Maule, que pertenecen a la Macrozona Centro y que registraban niveles de escorrentía per cápita de 444, 7.037 y 23.191 $\mathrm{m} 3 /$ persona/año, respectivamente. ${ }^{49}$

\footnotetext{
${ }^{47} \mathrm{El}$ promedio internacional se ha estimado en 1.700 m3/persona/año (sin estrés hídrico), según se indica en el Atlas del Agua de la DGA - 2016.

${ }^{48}$ Escorrentía media anual se define como "la estimación de todas las aguas que en forma efectiva, escurrirían superficialmente en una cuenca producto de las precipitaciones u otro aporte como media anual de largo plazo" (Atlas del Agua de la DGA - 2016).

${ }^{49}$ Valores entre 500 y 1.000 m3/persona/año (presión hídrica media), tienen efectos negativos para el desarrollo económico, salud y bienestar humano. Por su parte, valores menores a los $500 \mathrm{~m} 3 /$ persona/año (presión hídrica severa), en este
}

Esta situación, pone en evidencia la inconsistencia de fijar un mismo nivel de exigencia a los proyectos APR, mediante un valor de costo de referencia para una zona que agrupa 4 regiones del país, las cuales presentan diferencias significativas asociadas a las realidades hídricas que las afectan, tal como lo ha definido la DGA. escenario la presión hídrica es la principal restricción para la vida. 
Tabla 6: Elasticidad de la función TAP

Exponencial Hiperbólico un parametro Hiperbólico generalizado

\begin{tabular}{|c|c|c|c|c|c|c|c|c|c|c|c|}
\hline TSD & $\mathrm{N}^{\mathrm{o}} \mathrm{A}$ & TAP & ELAST. & TSD & $\mathrm{N}^{\mathrm{O}} \mathrm{A}$ & TAP & ELAST. & TSD & $\mathrm{N}^{\mathrm{O}} \mathrm{A}$ & TAP & ELAST. \\
\hline $0 \%$ & 12 & $28,6 \%$ & 0,00 & $0 \%$ & 12 & $28,6 \%$ & 0,00 & $0 \%$ & 12 & $28,6 \%$ & 0,00 \\
\hline $1 \%$ & 13 & $31,0 \%$ & 0,14 & $1 \%$ & 13 & $31,0 \%$ & 0,14 & $1 \%$ & 16 & $38,1 \%$ & 0,17 \\
\hline $2 \%$ & 16 & $38,1 \%$ & 0,27 & $2 \%$ & 16 & $38,1 \%$ & 0,27 & $2 \%$ & 17 & $40,5 \%$ & 0,06 \\
\hline $3 \%$ & 17 & $40,5 \%$ & 0,09 & $3 \%$ & 17 & $40,5 \%$ & 0,09 & $3 \%$ & 17 & $40,5 \%$ & 0,00 \\
\hline $4 \%$ & 17 & $40,5 \%$ & 0,00 & $4 \%$ & 17 & $40,5 \%$ & 0,00 & $4 \%$ & 17 & $40,5 \%$ & 0,00 \\
\hline $5 \%$ & 17 & $40,5 \%$ & 0,00 & $5 \%$ & 17 & $40,5 \%$ & 0,00 & $5 \%$ & 17 & $40,5 \%$ & 0,00 \\
\hline $6 \%$ & 17 & $40,5 \%$ & 0,00 & $6 \%$ & 17 & $40,5 \%$ & 0,00 & $6 \%$ & 17 & $40,5 \%$ & 0,49 \\
\hline $7 \%$ & 17 & $40,5 \%$ & 0,00 & $7 \%$ & 17 & $40,5 \%$ & 0,00 & $7 \%$ & 20 & $47,6 \%$ & 0,90 \\
\hline $8 \%$ & 17 & $40,5 \%$ & 0,44 & $8 \%$ & 17 & $40,5 \%$ & 0,00 & $8 \%$ & 22 & $52,4 \%$ & 0,56 \\
\hline $9 \%$ & 19 & $45,2 \%$ & 0,95 & $9 \%$ & 17 & $40,5 \%$ & 0,00 & $9 \%$ & 23 & $54,8 \%$ & 0,39 \\
\hline $10 \%$ & 21 & $50,0 \%$ & 0,95 & $10 \%$ & 17 & $40,5 \%$ & 0,00 & $10 \%$ & 24 & $57,1 \%$ & 0,21 \\
\hline $11 \%$ & 23 & $54,8 \%$ & 0,73 & $11 \%$ & 17 & $40,5 \%$ & 0,00 & $11 \%$ & 24 & $57,1 \%$ & 0,44 \\
\hline $12 \%$ & 24 & $57,1 \%$ & 0,73 & $12 \%$ & 17 & $40,5 \%$ & 0,67 & $12 \%$ & 26 & $61,9 \%$ & 0,48 \\
\hline $13 \%$ & 26 & $61,9 \%$ & 0,76 & $13 \%$ & 19 & $45,2 \%$ & 1,05 & $13 \%$ & 26 & $61,9 \%$ & 0,48 \\
\hline $14 \%$ & 27 & $64,3 \%$ & 0,52 & $14 \%$ & 20 & $47,6 \%$ & 0,70 & $14 \%$ & 28 & $66,7 \%$ & 0,52 \\
\hline $15 \%$ & 28 & $66,7 \%$ & 0,27 & $15 \%$ & 21 & $50,0 \%$ & 0,71 & $15 \%$ & 28 & $66,7 \%$ & 0,00 \\
\hline $16 \%$ & 28 & $66,7 \%$ & 0,00 & $16 \%$ & 22 & $52,4 \%$ & 0,73 & $16 \%$ & 28 & $66,7 \%$ & 0,00 \\
\hline $17 \%$ & 28 & $66,7 \%$ & 0,00 & $17 \%$ & 23 & $54,8 \%$ & 0,38 & $17 \%$ & 28 & $66,7 \%$ & 0,00 \\
\hline $18 \%$ & 28 & $66,7 \%$ & 0,00 & $18 \%$ & 23 & $54,8 \%$ & 0,38 & $18 \%$ & 28 & $66,7 \%$ & 0,00 \\
\hline $19 \%$ & 28 & $66,7 \%$ & 0,33 & $19 \%$ & 24 & $57,1 \%$ & 0,40 & $19 \%$ & 28 & $66,7 \%$ & 0,00 \\
\hline $20 \%$ & 29 & $69,0 \%$ & 0,35 & $20 \%$ & 24 & $57,1 \%$ & 0,00 & $20 \%$ & 28 & $66,7 \%$ & 0,35 \\
\hline
\end{tabular}

No A : Número de proyectos aprobados; ELAST.: Nivel de elasticidad de la TAP frente a cambios en el valor de TSD Fuente: Elaboración propia. 
Tabla 7: Diferencia del costo de referencia de la zona 3

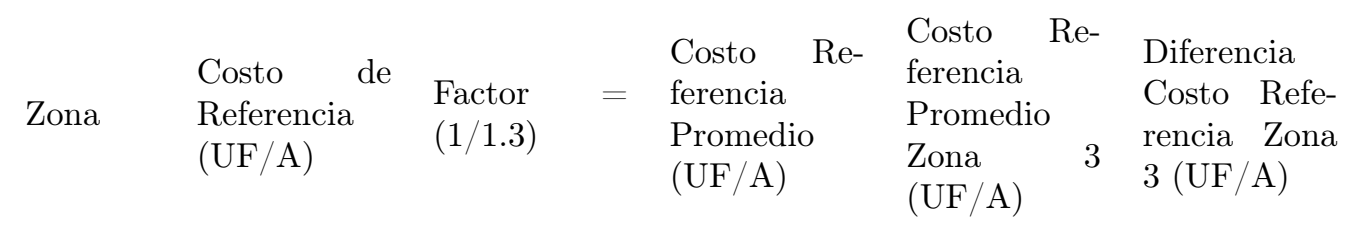

\begin{tabular}{llllll} 
& $(\mathrm{A})$ & $(\mathrm{B})$ & $\begin{array}{l}(\mathrm{C}) \\
(\mathrm{A}) *(\mathrm{~B})\end{array}$ & $(\mathrm{D})$ & $\begin{array}{l}(\mathrm{E})=(\mathrm{C}- \\
\mathrm{D})\end{array}$ \\
\hline 1 & 230 & 0.769 & 177 & 111 & 66 \\
2 & 221 & 0.769 & 170 & 111 & 59 \\
3 & 144 & 0.769 & 111 & 111 & 0 \\
4 & 175 & 0.769 & 135 & 111 & 24 \\
5 & 213 & 0.769 & 164 & 111 & 53 \\
6 & 163 & 0.769 & 125 & 111 & 14 \\
\hline \multicolumn{5}{c}{ UF/A Fuente: Elaboración propia. } \\
\end{tabular}

Figura 7: Escorrentía y escorrentía per cápita a nivel nacional distribuidos por regiones

\begin{tabular}{|c|c|c|c|c|c|}
\hline Macrozona & Región & Escorrentía & {$\left[\mathrm{m}^{3} / \mathrm{s}\right]$} & [\%] & Escorrentía per cápita (2015) [m³/persona/año] \\
\hline \multirow{5}{*}{ Norte } & $x v$ & & 5,5 & 0,019 & 725 \\
\hline & 1 & & 6,4 & 0,022 & 599 \\
\hline & $\|$ & & 0,9 & 0,003 & 47 \\
\hline & III & & 1,9 & 0,006 & 190 \\
\hline & IV & & 22,2 & 0,08 & 908 \\
\hline \multirow{4}{*}{ Centro } & $\mathrm{v}$ & & 41 & 0,14 & 703 \\
\hline & RM & I & 103 & 0,35 & 444 \\
\hline & v & I & 205 & 0,7 & 7.037 \\
\hline & VII & 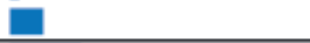 & 767 & 2,62 & 23.191 \\
\hline \multirow{4}{*}{ Sur } & VIII & & 1.638 & 5,6 & 24.432 \\
\hline & $\mathrm{IX}$ & $\square$ & 1.041 & 3,56 & 33.167 \\
\hline & XIV & $\square$ & 1.046 & 3,58 & 81.563 \\
\hline & $\mathrm{x}$ & & 4.109 & 14,05 & 154.058 \\
\hline \multirow{3}{*}{ Austral } & $\mathrm{xI}$ & & 10.134 & 34,65 & 2.950 .168 \\
\hline & XII & & 10.124 & 34,62 & 1.938 .956 \\
\hline & & Total & 29.245 & & 51.218 \\
\hline
\end{tabular}

Fuente: Dirección General de Aguas (DGA - 2016). 
Tabla 8: Criterio TDLA y elasticidad de las 6 Zonas del país

Criterio de decisión aprobar proyectos con TDLA menor o igual a TSD $=6 \%$

\begin{tabular}{|c|c|c|c|c|c|c|}
\hline Decisión & Zona 1 & Zona 2 & Zona 3 & Zona 4 & Zona 5 & Zona 6 \\
\hline № Proy. Aprobados & 29 & 28 & 17 & 23 & 27 & 22 \\
\hline № Proy. Rechazados & 13 & 14 & 25 & 19 & 15 & 20 \\
\hline \multicolumn{7}{|c|}{ Valor de Elasticidad función Tasa de Aprobación Proyectos (TAP) } \\
\hline TSD & Zona 1 & Zona 2 & Zona 3 & Zona 4 & Zona 5 & Zona 6 \\
\hline $0 \%$ & 0,00 & 0,00 & 0,00 & 0,00 & 0,00 & 0,00 \\
\hline $1 \%$ & 0,07 & 0,10 & 0,14 & 0,00 & 0,10 & 0,09 \\
\hline $2 \%$ & 0,09 & 0,09 & 0,27 & 0,11 & 0,10 & 0,00 \\
\hline $3 \%$ & 0,25 & 0,13 & 0,09 & 0,23 & 0,07 & 0,23 \\
\hline $4 \%$ & 0,24 & 0,24 & 0,00 & 0,19 & 0,33 & 0,31 \\
\hline $5 \%$ & 0,27 & 0,38 & 0,00 & 0,23 & 0,40 & 0,12 \\
\hline $6 \%$ & 0,32 & 0,33 & 0,00 & 0,26 & 0,22 & 0,27 \\
\hline $7 \%$ & 0,23 & 0,24 & 0,00 & 0,29 & 0,37 & 0,30 \\
\hline $8 \%$ & 0,26 & 0,27 & 0,44 & 0,47 & 0,28 & 0,33 \\
\hline $9 \%$ & 0,14 & 0,29 & 0,95 & 0,51 & 0,15 & 0,53 \\
\hline $10 \%$ & 0,15 & 0,16 & 0,95 & 0,18 & 0,32 & 0,57 \\
\hline $11 \%$ & 0,33 & 0,33 & 0,73 & 0,19 & 0,17 & 0,20 \\
\hline $12 \%$ & 0,35 & 0,36 & 0,73 & 0,21 & 0,36 & 0,21 \\
\hline $13 \%$ & 0,19 & 0,19 & 0,76 & 0,22 & 0,39 & 0,23 \\
\hline $14 \%$ & 0,00 & 0,20 & 0,52 & 0,24 & 0,20 & 0,24 \\
\hline $15 \%$ & 0,00 & 0,00 & 0,27 & 0,25 & 0,22 & 0,25 \\
\hline $16 \%$ & 0,00 & 0,00 & 0,00 & 0,26 & 0,00 & 0,00 \\
\hline $17 \%$ & 0,00 & 0,00 & 0,00 & 0,00 & 0,00 & 0,00 \\
\hline $18 \%$ & 0,25 & 0,00 & 0,00 & 0,00 & 0,00 & 0,30 \\
\hline $19 \%$ & 0,27 & 0,27 & 0,33 & 0,88 & 0,00 & 0,31 \\
\hline $20 \%$ & 0,00 & 0,28 & 0,35 & 0,92 & 0,28 & 0,00 \\
\hline
\end{tabular}

Fuente: Elaboración propia. 
Tabla 9: Zona 3 con cambios en el valor costo de referencia Criterio de decisión aprobar proyectos con TDLA menor o igual a TSD =6\%

\begin{tabular}{|c|c|c|c|c|c|c|}
\hline Decisión & Zona 1 & Zona 2 & Zona 3 & Zona 4 & Zona 5 & Zona 6 \\
\hline № Proy. Aprobados & 29 & 28 & 22 & 23 & 27 & 22 \\
\hline № Proy. Rechazados & 13 & 14 & 20 & 19 & 15 & 20 \\
\hline \multicolumn{7}{|c|}{ Valor de Elasticidad función Tasa de Aprobación Proyectos (TAP) } \\
\hline TSD & Zona 1 & Zona 2 & Zona 3 & Zona 4 & Zona 5 & Zona 6 \\
\hline $0 \%$ & 0,00 & 0,00 & 0,00 & 0,00 & 0,00 & 0,00 \\
\hline $1 \%$ & 0,07 & 0,10 & 0,03 & 0,00 & 0,10 & 0,09 \\
\hline $2 \%$ & 0,09 & 0,09 & 0,00 & 0,11 & 0,10 & 0,00 \\
\hline $3 \%$ & 0,25 & 0,13 & 0,17 & 0,23 & 0,07 & 0,23 \\
\hline $4 \%$ & 0,24 & 0,24 & 0,22 & 0,19 & 0,33 & 0,31 \\
\hline $5 \%$ & 0,27 & 0,38 & 0,37 & 0,23 & 0,40 & 0,12 \\
\hline $6 \%$ & 0,32 & 0,33 & 0,57 & 0,26 & 0,22 & 0,27 \\
\hline $7 \%$ & 0,23 & 0,24 & 0,45 & 0,29 & 0,37 & 0,30 \\
\hline $8 \%$ & 0,26 & 0,27 & 0,64 & 0,47 & 0,28 & 0,33 \\
\hline $9 \%$ & 0,14 & 0,29 & 0,51 & 0,51 & 0,15 & 0,53 \\
\hline $10 \%$ & 0,15 & 0,16 & 0,18 & 0,18 & 0,32 & 0,57 \\
\hline $11 \%$ & 0,33 & 0,33 & 0,00 & 0,19 & 0,17 & 0,20 \\
\hline $12 \%$ & 0,35 & 0,36 & 0,00 & 0,21 & 0,36 & 0,21 \\
\hline $13 \%$ & 0,19 & 0,19 & 0,23 & 0,22 & 0,39 & 0,23 \\
\hline $14 \%$ & 0,00 & 0,20 & 0,48 & 0,24 & 0,20 & 0,24 \\
\hline $15 \%$ & 0,00 & 0,00 & 0,25 & 0,25 & 0,22 & 0,25 \\
\hline $16 \%$ & 0,00 & 0,00 & 0,00 & 0,26 & 0,00 & 0,00 \\
\hline $17 \%$ & 0,00 & 0,00 & 0,28 & 0,00 & 0,00 & 0,00 \\
\hline $18 \%$ & 0,25 & 0,00 & 0,30 & 0,00 & 0,00 & 0,30 \\
\hline $19 \%$ & 0,27 & 0,27 & 0,30 & 0,88 & 0,00 & 0,31 \\
\hline $20 \%$ & 0,00 & 0,28 & 0,32 & 0,92 & 0,28 & 0,00 \\
\hline
\end{tabular}

Fuente: Elaboración propia. 


\subsection{Impacto en la función TAP respecto de políticas públicas}

Se desarrollan tres simulaciones que consisten en incorporar mayores niveles de recursos en los 42 proyectos con el objeto de dar solución a los principales problemas que presentan los sistemas APR. ${ }^{50}$ Dentro de los problemas que se destacan, estos corresponden a i) Mayor costo de solución por arranque, ii) Deficiencias de diseño e innovación de los sistemas y iii) Deficiencias en la gestión de los sistemas APR.

El procedimiento adoptado en cada simulación consiste en comparar la situación inicial, asociada a la cantidad aprobada de proyectos definida para cada nivel de $\mathrm{TSD}^{51}$ (ver anexo 2) versus la cantidad aprobada de proyectos al incluir cambios exógenos en los perfiles de los costos de los 42 proyectos APR. Estas pruebas son realizadas para cada factor de descuento (exponencial e hiperbólico) y buscan determinar el impacto de estos cambios en la TAP.

La primera simulación será definida como caso 1, que consiste en incrementar el valor de la inversión inicial en un monto igual al $7 \%$ del ítem asociado a los costos de distribución de los sistemas APR. Este incremento corresponde al promedio anual que la

\footnotetext{
${ }^{50}$ Según se desprende del "Informe Final de Evaluación de Infraestructura de Agua Potable Rural" (Dirección de Obras Hidráulicas, 2015), de la DOH.

${ }^{51}$ Se aplicó el criterio de decisión consistente en aprobar aquellos proyectos que registren una TDLA menor o igual para determinado nivel de TSD.
}

DOH ha estimado como crecimiento para el periodo 2011-2014. Esta simulación se orienta a dar solución al primer problema enunciado por la DOH, que corresponde al mayor costo de solución por arranque.

La segunda simulación será definida como caso 2, que incorpora el periodo 10 (diez), un monto equivalente al $25 \%$ del valor total de los costos de operación, mantención y administración de los sistemas APR. ${ }^{52}$ Este incremento será variable para cada proyecto, ya que algunos si aplican dicho valor y otros en un porcentaje menor. El monto queda representado dentro de la estructura de costos del proyecto en el ítem costo de reposición de los sistemas APR. Esta simulación se orienta a dar solución al tercer problema enunciado por la $\mathrm{DOH}$, asociado a deficiencias en la gestión de los sistemas APR.

La tercera simulación será definida como caso 3, que consiste en incrementar simultáneamente con los valores establecidos para las simulaciones de los casos 1 y 2, los montos de inversión inicial y flujo de los costos del proyecto (periodo 10). Esta simulación busca observar el impacto de dichas medidas en la solución de los 2 problemas enunciados anteriormente y como se comportan los resultados en la TAP al realizar el ejercicio en forma simultánea.

En la anexo 4 se presentan los resultados para cada una de las simulaciones realizadas, donde es posible observar valores en color azul y rojo, ${ }^{53}$ que indican la variación en la cantidad aprobada de proyectos, con respecto a la situación inicial indicada en el anexo 2. Para el caso 1, se observa que para valores de TSD iguales al 5 y $6 \%$, se mantiene el nivel de TAP en un 40.5\% (se aprueban 17 de los 42 proyectos), independiente del factor de descuento utilizado en la evaluación económica. Ello sugiere que la implementación de proyectos APR por parte del Estado, producto del menor costo de financiar dichos proyectos (menor TSD), solo tiene efectos en el mayor gasto en inversión a ser realizado por parte del Estado (financiar sistemas APR más caros).

\footnotetext{
${ }^{52}$ Este incremento viene en relación a la aplicación óptima de las tarifas por suministro de servicio de agua potable rural, las cuales bajo la normativa ley 20.998 deben asegurar el financiamiento de los gastos necesarios para la mantención y reparación de los sistemas, como así también fondos a destinar a planes de reinversión.

${ }^{53} \mathrm{El}$ valor cero en color azul indica que no existe variación en la TAP (número de proyectos aprobados) para cada nivel de TSD producto de cambios en algunos de los ítems del proyecto (caso 1, 2 y 3 ). Por su parte, los valores en color rojo indica variación negativa en la TAP (cantidad de proyectos rechazados) para cada nivel de TSD, al ser comparado con la situación inicial de la TAP (anexo 2).
} 
En el caso 2, se observa que para un valor de TSD igual al $6 \%$ se mantiene el nivel de TAP igual al $40.5 \%$ (se aprueban 17 de los 42 proyectos), tanto para evaluaciones económicas realizadas con uso del factor de descuento exponencial e hiperbólico generalizado. En cuanto al factor hiperbólico de un parámetro, se observa una disminución en el nivel de TAP, pasando del 40.5 al $38.1 \%$ (de 17 a 16 proyectos aprobados). Por su parte, en un escenario de disminución en el valor de la TSD del 6 al $5 \%$, el factor de descuento hiperbólico generalizado es el único que mantiene el nivel inicial de TAP (40.5\%), registrando los otros factores de descuento disminución en la TAP.

Por lo tanto, políticas públicas orientadas a incrementar las tarifas en el porcentaje y cantidad exigidas bajo el nuevo marco regulatorio, aplicadas en un escenario de TSD en disminución, una evaluación económica realizada con uso del factor de descuento exponencial (tradicional) e hiperbólico de un parámetro, disminuyen la cantidad aprobada de proyectos. Sin embargo, para el caso del factor de descuento hiperbólico generalizado, se mantiene la cantidad de proyectos aprobados (TAP igual a $40.5 \%$ ).

En el caso 3, se observa que para un valor de TSD igual al $6 \%$, el único factor de descuento que mantiene la TAP inicial de $40.5 \%$ es el factor hiperbólico generalizado. El factor exponencial registra una disminución en la TAP, pasando del 40.5 al $38.1 \%$ (17 a 16 proyectos aprobados) y el factor hiperbólico de un parámetro, disminuye del 40.5 al $28.6 \%$ (17 a 12 proyectos aprobados). Por su parte, frente a un escenario de disminución en el valor de la TSD del 6 al $5 \%$, el factor hiperbólico generalizado presenta ventaja en su uso, ya que es el único que mantiene la TAP inicial $(40.5 \%)$, registrando los otros factores de descuentos significativas disminuciones en la TAP.

Por lo tanto, la implementación en forma simultánea de incrementos en los niveles de inversión inicial (mayor costo por solución de los sistemas APR) y flujo de los costos del proyecto (inversión en reposición de los sistemas); indican que, en un contexto de TSD en disminución, evaluaciones económicas realizadas con factor de descuento hiperbólico generalizado se obtienen los mejores resultados, ya que no tienen efectos en la TAP.

Ahora bien, conforme la realidad presente en los sistemas APR del país y en cuanto a las simulaciones realizadas anteriormente, el caso 3 se adecua de mejor forma a la situación que el sector de agua po- table rural deberá enfrentar en el futuro, es decir, mayor costo de solución de los sistemas y exigencias en el control de las tarifas de agua potable, conforme lo indica la nueva normativa.

En consideración a ello, se plantea una prueba la cual será definida como caso $3(\mathrm{~N})$, la cual se diferencia de la prueba 3 antes descrita, en que adicionalmente a los cambios realizados en los montos de inversión inicial y flujo de los costos, se introducen cambios en el valor del costo de referencia. Con ello, se evalúa la variación en la TAP para cada nivel de TSD, con respecto a los valores obtenidos en la situación inicial (anexo 2).

En cuanto a los cambios al valor de los costos de referencia, estos consisten en reemplazar de 130 a 141 UF para aquellos proyectos aprobados en el periodo 2012-2014 y de 144 a 152 UF para aquellos proyectos aprobados en el periodo 2015-2017. La razón por la cual se considera este ajuste, responde a los resultados obtenidos en el análisis de estabilidad de los datos de los proyectos (ver sección 4.3), donde se sugieren realizar ajustes al alza en su valor, debido a que permiten dar consistencia a los resultados económicos obtenidos para los 42 proyectos APR de la región del Bio Bio al ser estos evaluados en distintas zonas del país. ${ }^{54}$

En el anexo 5 se indican los resultados para el caso $3(\mathrm{~N})$, donde es posible observar que todos los factores de descuentos (exponencial e hiperbólicos) aumentan la cantidad de proyectos aprobados (números en color verde) para determinados niveles de TSD. Además, se observa que para valores de TSD igual al 5 y $6 \%$, con uso de factores de descuento exponencial e hiperbólico de un parámetro, se mantiene la TAP en un valor igual al 40.5\% (se aprueban 17 de los 42 proyectos) y con uso del factor hiperbólico generalizado se aumenta la TAP pasando del 40.5 al $42.9 \%$, es decir, se aumenta de 17 a 18 proyectos aprobados.

Por lo tanto, políticas públicas que se orienten al fomento de iniciativas que permitan desarrollar la instalación de sistemas APR en la región del Bio Bio, producto del menor costo de los fondos para el país (menor valor TSD), sin perjuicio del incrementos en el valor de los costos de los proyectos APR (según los montos indicados para los casos 1 y 2); como así también, ajustes al alza en el valor del costo de referencia de la zona 3; evaluaciones económicas

\footnotetext{
${ }^{54}$ Los resultados económicos consistentes dicen relación los valores obtenidos en la TAP y la elasticidad de la función TAP.
} 
realizadas con uso de factor de descuento hiperbólico generalizado se obtienen los mejores resultados, ya que para un rango de TSD del 3 al $6 \%$ se obtienen incrementos en la TAP (del $40.5 \%$ al $42.9 \%$ ). Sin embargo, los factores de descuento exponencial (tradicional) e hiperbólico de un parámetro, mantienen o disminuyen la TAP.

\section{Conclusiones}

Sobre la base de 42 proyectos de Agua Potable Rural (APR) pertenecientes a la región del Bio Bio, esta investigación permitió observar que mediante el uso de indicadores económicos basados en el enfoque Costo Eficiencia, independiente del factor de descuento utilizado en una evaluación económica, sean estos; el factor exponencial (tradicional) o factores de descuento basados en funciones hiperbólicas; políticas públicas que se orienten a incentivar la instalación de los sistemas APR en un contexto de disminución en el valor de la TSD, los resultados indican que los niveles de Tasa de Aprobación de los Proyectos (TAP) no se ven afectados, toda vez la elasticidad en la TAP para un rango de TSD del 6 al $5 \%$ son iguales a cero (nula).

En cuanto a la implementación del nuevo marco regulatorio de agua potable rural, los resultados indican que bajo un escenario que incluya mayores costos de solución de los sistemas APR, control adecuado en la fijación y recaudación de tarifas por suministro de agua potable y ajustes al alza en el valor del costo de referencia de los proyectos APR, evaluaciones económicas realizadas con uso del factor de descuento hiperbólico generalizado, aplicadas en un contexto de TSD en disminución (menor al 6\%), se logran los mejores resultados, toda vez aumenta la cantidad de proyectos aprobados.

Además, esta investigación permitió analizar el proceso de evaluación económica de los proyectos APR realizada por el Ministerio de Desarrollo Social (MDS), donde se logro observar la importancia dada en la evaluación económica a los requisitos de factibilidad técnica de los proyectos APR, representados por el costo de referencia por solución para un sistema APR, por sobre la evaluación económica de diversas alternativas de solución de suministro de agua potable, las que se evalúan por medio de los indicadores económicos Valor Actual de los Costos (VAC) y Costo Anual Equivalente (CAE), los que se basan en el enfoque Costo Eficiencia.

También es posible observar que, conforme las he- rramientas suministradas por la evaluación económica de los proyectos y considerando en el análisis económico el costo de referencia como aquel beneficio mínimo que el MDS exige a los proyectos APR necesarios para dar la categoría de Recomendación Sin Condiciones (RS) y de este modo aprobar su ejecución, decisiones basadas en el uso de la Tasa de Descuento Límite de Aprobación (TDLA) de los proyectos, sugerida en esta investigación, se registra una menor cantidad de proyectos aprobados por este procedimiento versus la cantidad aprobada bajo el criterio de decisión basado en el costo de referencia.

Finalmente, mediante los procesos aplicados para la estimación de la TDLA y elasticidad de la TAP, permitieron encontrar observaciones al valor del costo de referencia que es registrado como límite de aprobación de los proyectos APR de la región del Bio Bio. Lo anterior, se logra evidenciar por medio de las pruebas de estabilidad desarrolladas en esta investigación, las que sugieren como conclusión que para la muestra de 42 proyectos APR evaluados en las 6 zonas del país, conforme la definición geográfica establecida por el MDS, la zona 3 en la cual está inserta la región del Bio Bio, presenta diferencias significativas en costos con respecto a las otras 5 zonas del país, lo que permiten sugerir que el costo de referencia que fija el MDS para la zona 3, no está bien ajustado.

\section{Referencias}

Campos, J., Serebrisky, T., y Suárez-Alemán, A. (2015). Porque el tiempo pasa: evolución teórica y práctica en la determinación de la tasa social de descuento. Banco Interamericano de Desarrollo.

Campos, J., Serebrisky, T., y Suárez-Alemán, A. (2016). Tasa de descuento social y evaluación de proyectos: Algunas reflexione prácticas para América Latina y el Caribe. Banco Interamericano de Desarrollo.

Cartes, F., Contreras, E., y Cruz, J. M. (2004). La tasa social de descuento en Chile. Facultad de Ciencias de la Ingeniería, Universidad de Chile, Santiago de Chile.

Correa, R. (2006). La tasa social de descuento y el medio ambiente. Lecturas de Economía, 64:91116.

Dirección de Obras Hidráulicas (2015). Informe Final de Evaluación de Infraestructura Hidráulica de Agua Potable Rural (APR). Dirección de 
Obras Hidráulicas, Ministerio de Obras Públicas, Chile, Santiago de Chile.

Edwards, G. (2016). Tasa social de descuento en el largo plazo en el marco de los sistemas nacionales de inversiones en chile: aplicación al caso de chileno. El Trimestre Económico, LXXXIII(329):99-125.

Harberger, A. (1972). On Measuring the Social Opportunity Cost of Public Funds. The University of Chicago Press, Chicago.

Homsi y Asociados Ltda (2007). Estudio de Saneamiento Rural. KRISTAL Ingeniería Ambiental.

Laibson, D. (1997). Golden eggs and hyperbolic discounting. Quarterly Journal of Economics, 112:443-477.

Mazur, J. E. (1987). An adjusting procedure for studying delayed reinforcement. En Commons, M. L., Mazur, J. E., Nevin, J. A., y Rachlin, H., editores, The Effect of Delay and of Intervening Events on Reinforcement Value, Quantitative Analyses of Behavior, pp. 55-73. Erlbaum," New Jersey.

Oddene, G. (2011). Componente: "Tasa Social de Descuento" Convenio OPP - FCEyA. Facultad de Ciencias Económicas y Administrativas, Universidad de la Republica, Montevideo.

Poulos, C. y Whittington, D. (2000). Time preferences for life-saving programs: evidence from six less developed countries. ACS Publications.

Romano, G. (2014). Decisiones intertemporales y decisiones bajo incertidumbre: relaciones lógicas y conceptuales. Número 1.

Samuelson, P. A. (1937). A note on measurement of utility. The Review of Economic Studies, $4(2): 155-161$.

Rev. Est. de Políticas Públicas, 5(2): julio 2019 - noviembre 2019 


\section{Anexo 1: Nómina de proyectos APR en estudio}

\begin{tabular}{|c|c|c|c|c|c|c|c|}
\hline $\begin{array}{c}\mathrm{N}^{\circ} \\
\text { Proyecto } \\
\end{array}$ & Nombre Sistema APR & Provincia & Tipo de Proyecto & \begin{tabular}{c|}
$\begin{array}{c}\text { Inversión } \\
\text { UF }\end{array}$ \\
\end{tabular} & Año & \begin{tabular}{|c|}
$\mathrm{N}^{\circ}$ \\
Arranques \\
\end{tabular} & \begin{tabular}{|c|}
$\mathrm{N}^{\circ}$ \\
Beneficiarios \\
\end{tabular} \\
\hline 1 & Tirúa & Arauco & Mantención y Ampliación & 176.487 & 2.015 & 1.139 & 5.435 \\
\hline 2 & El Zarzal - Monteblanco - Calle Dávila & Nuble & Construccion APR & 23.038 & 2.014 & 164 & 810 \\
\hline 3 & San Antonio - Las Quintas - Allipen & Bio-Bio & Construccion APR & 43.162 & 2.014 & 278 & 1.345 \\
\hline 4 & San José de Bío Bín & Bio-Bio & Construccion APR & 14.773 & 2.015 & 75 & 415 \\
\hline 5 & San Jose-Las Achiras & Nuble & Construccion APR & 29.398 & 2.016 & 108 & 413 \\
\hline 6 & San Pedro -San Ignacio & Nuble & Construccion APR & 16.702 & 2.013 & 71 & 351 \\
\hline 7 & Santa Amelia & Bio-Bio & Construccion APR & 20.596 & 2.017 & 116 & 569 \\
\hline 8 & Santa Cruz de Cuca & Ñuble & Construccion APR & 20.376 & 2.012 & 183 & 880 \\
\hline 9 & Santa Juana-El Calvario & Nuble & Mantención y Ampliación & 34.246 & 2.016 & 281 & 1.147 \\
\hline 10 & Selva Negra & Nuble & Construccion APR & 22.038 & 2.015 & 112 & 520 \\
\hline 11 & Mesamavida & $\mathrm{Bio}-\mathrm{Bio}$ & Construccion APR & 20.575 & 2.017 & 131 & 653 \\
\hline 12 & Oro Verde & Nuble & Construccion APR & 38.900 & 2.015 & 223 & 1.825 \\
\hline 13 & Pata de Gallina & Bio-Bio & Construccion APR & 17.716 & 2.012 & 131 & 460 \\
\hline 14 & Peñaflor & Bio-Bio & Construccion APR & 51.587 & 2.015 & 321 & 1.565 \\
\hline 15 & Pichiarauco - Vvilla los ríos & Arauco & Construccion APR & 49.749 & 2.014 & 237 & 1.145 \\
\hline 16 & Pilicura & Nuble & Construccion APR & 17.244 & 2.014 & 86 & 430 \\
\hline 17 & Poco a poco & Bio-Bio & Construccion APR & 11.419 & 2.016 & 109 & 436 \\
\hline 18 & Quinquehua & Nuble & Mantención y Ampliación & 17.479 & 2.014 & 270 & 640 \\
\hline 19 & Relbún & Nuble & Construccion APR & 30.292 & 2.014 & 168 & 832 \\
\hline 20 & Rinconada de Itata & Nuble & Construccion APR & 11.732 & 2.013 & 70 & 345 \\
\hline 21 & Las Vegas & $\mathrm{Bin}-\mathrm{Bio}$ & Construccion APR & 18.590 & 2.016 & 183 & 732 \\
\hline 22 & Las Vertientes & Nuble & Construccion APR & 26.068 & 2.015 & 159 & 795 \\
\hline 23 & Llano Blanco & Bio-Bio & Construccion APR & 40.431 & 2.013 & 267 & 1.270 \\
\hline 24 & Los Boldos - Mañil Bajo - Los Naranjos & Bio-Bio & Construccion APR & 58.917 & 2.013 & 509 & 2.382 \\
\hline 25 & La Curva & Arauco & Construccion APR & 27.932 & 2.016 & 199 & 538 \\
\hline 26 & La Isla & $\mathrm{Bio}-\mathrm{Bio}$ & Construccion APR & 22.112 & 2.017 & 121 & 605 \\
\hline 27 & La Mata - Las Garzas & Nuble & Construccion APR & 23.942 & 2.017 & 140 & 417 \\
\hline 28 & La Perla & Bio-Bio & Construccion APR & 51.942 & 2.016 & 359 & 1.233 \\
\hline 29 & Laraquete - El Pinar & Arauco & Mantención y Ampliación & 32.823 & 2.016 & 1.448 & 6.323 \\
\hline 30 & Las aguilas & Bio-Bio & Construccion APR & 14.334 & 2.016 & 93 & 421 \\
\hline 31 & Elaromo & Bio-Bio & Construccion APR & 45.837 & 2.017 & 241 & 944 \\
\hline 32 & El olivo & Bio-Bio & Construccion APR & 18.184 & 2.016 & 146 & 587 \\
\hline 33 & Elpino san miguel & Bio-Bio & Construccion APR & 35.200 & 2.014 & 217 & 1.050 \\
\hline 34 & Frutillares & Nuble & Construccion APR & 19.785 & 2.014 & 117 & 555 \\
\hline 35 & Horcones & Arauco & Construccion APR & 21.963 & 2.012 & 372 & 1.735 \\
\hline 36 & Junquillo & Nuble & Construccion APR & 13.685 & 2.016 & 84 & 252 \\
\hline 37 & La Gloria & Concepción & Construccion APR & 15.853 & 2.017 & 77 & 291 \\
\hline 38 & Cantarrana & Bio-Bio & Construccion APR & 23.398 & 2.015 & 134 & 610 \\
\hline 39 & Ciruelito - ElCardal & Ñuble & Construccion APR & 21.512 & 2.014 & 156 & 745 \\
\hline 40 & Coleal Central & Nuble & Construccion APR & 16.071 & 2.014 & 123 & 585 \\
\hline 41 & Dicahue & $\mathrm{Bio}-\mathrm{Bin}$ & Construccion APR & 32.132 & 2.016 & 221 & 774 \\
\hline 42 & Diuto & Bio-Bio & Construccion APR & 14.928 & 2.013 & 134 & 650 \\
\hline Obs. 1 & \multicolumn{7}{|c|}{ El monto de la inversión se asocia al valor a ser ejecutado según presupuesto y no al valor asociado a la evaluación económica. } \\
\hline Obs. 2 & \multicolumn{7}{|c|}{ El año hace referencia al periodo donde fue aprobada la evaluación económica del proyecto. } \\
\hline Obs. 3 & \multicolumn{7}{|c|}{ El número de Arranques y Beneficiarios corresponde al proyecto en marcha y no al considerado en la evalua ción económica. } \\
\hline
\end{tabular}




\section{Anexo 2: Tasa de Aprobación de Proyectos}

\begin{tabular}{|c|c|c|c|c|c|c|}
\hline \multicolumn{7}{|c|}{$\begin{array}{c}\text { Número y Porcentaje de Proyectos APR Aprobados para cada nivel de TSD } \\
\text { Muestra de } 42 \text { proyectos APR Región del Bio Bio }\end{array}$} \\
\hline \multirow{2}{*}{ TSD } & \multicolumn{2}{|c|}{ Exponencial } & \multicolumn{2}{|c|}{ Hiperbólico un Parámetro } & \multicolumn{2}{|c|}{ Hiperbólico Gener alizado } \\
\hline & $\mathrm{N}^{\circ}$ Aprobados & $(\%)$ Muestra & $\mathrm{N}^{\circ}$ Aprobados & $(\%)$ Muestra & $\mathrm{N}^{\circ}$ Aprobados & (\%)Muestra \\
\hline TSD igual a $0 \%$ & 12 & $28,6 \%$ & 12 & $28,6 \%$ & 12 & $28,6 \%$ \\
\hline TSD igual a $1 \%$ & 13 & $31,0 \%$ & 13 & $31,0 \%$ & 16 & $38,1 \%$ \\
\hline TSD igual a $2 \%$ & 16 & $38,1 \%$ & 16 & $38,1 \%$ & 17 & $40,5 \%$ \\
\hline TSD igual a $3 \%$ & 17 & $40,5 \%$ & 17 & $40,5 \%$ & 17 & $40,5 \%$ \\
\hline TSD igual a $4 \%$ & 17 & $40,5 \%$ & 17 & $40,5 \%$ & 17 & $40,5 \%$ \\
\hline TSD igual a 5\% & 17 & $40,5 \%$ & 17 & $40,5 \%$ & 17 & $40,5 \%$ \\
\hline TSD igual a $6 \%$ & 17 & $40,5 \%$ & 17 & $40,5 \%$ & 17 & $40,5 \%$ \\
\hline TSD igual a $7 \%$ & 17 & $40,5 \%$ & 17 & $40,5 \%$ & 20 & $47,6 \%$ \\
\hline TSD igual a $8 \%$ & 17 & $40,5 \%$ & 17 & $40,5 \%$ & 22 & $52,4 \%$ \\
\hline TSD igual a $9 \%$ & 19 & $45,2 \%$ & 17 & $40,5 \%$ & 23 & $54,8 \%$ \\
\hline TSD igual a $10 \%$ & 21 & $50,0 \%$ & 17 & $40,5 \%$ & 24 & $57,1 \%$ \\
\hline TSD igual a $11 \%$ & 23 & $54,8 \%$ & 17 & $40,5 \%$ & 24 & $57,1 \%$ \\
\hline TSD igual a $12 \%$ & 24 & $57,1 \%$ & 17 & $40,5 \%$ & 26 & $61,9 \%$ \\
\hline TSD igual a $13 \%$ & 26 & $61,9 \%$ & 19 & $45,2 \%$ & 26 & $61,9 \%$ \\
\hline TSD igual a $14 \%$ & 27 & $64,3 \%$ & 20 & $47,6 \%$ & 28 & $66,7 \%$ \\
\hline TSD igual a $15 \%$ & 28 & $66,7 \%$ & 21 & $50,0 \%$ & 28 & $66,7 \%$ \\
\hline TSD igual a $16 \%$ & 28 & $66,7 \%$ & 22 & $52,4 \%$ & 28 & $66,7 \%$ \\
\hline TSD igual a $17 \%$ & 28 & $66,7 \%$ & 23 & $54,8 \%$ & 28 & $66,7 \%$ \\
\hline TSD igual a $18 \%$ & 28 & $66,7 \%$ & 23 & $54,8 \%$ & 28 & $66,7 \%$ \\
\hline TSD igual a $19 \%$ & 28 & $66,7 \%$ & 24 & $57,1 \%$ & 28 & $66,7 \%$ \\
\hline TSD igual a $20 \%$ & 29 & $69,0 \%$ & 24 & $57,1 \%$ & 28 & $66,7 \%$ \\
\hline TSD igual a $21 \%$ & 29 & $69,0 \%$ & 24 & $57,1 \%$ & 29 & $69,0 \%$ \\
\hline TSD igual a $22 \%$ & 29 & $69,0 \%$ & 24 & $57,1 \%$ & 29 & $69,0 \%$ \\
\hline TSD igual a $23 \%$ & 29 & $69,0 \%$ & 25 & $59,5 \%$ & 30 & $71,4 \%$ \\
\hline TSD igual a $24 \%$ & 29 & $69,0 \%$ & 26 & $61,9 \%$ & 30 & $71,4 \%$ \\
\hline TSD igual a $25 \%$ & 29 & $69,0 \%$ & 26 & $61,9 \%$ & 30 & $71,4 \%$ \\
\hline TSD igual a $26 \%$ & 29 & $69,0 \%$ & 26 & $61,9 \%$ & 30 & $71,4 \%$ \\
\hline TSD igual a $27 \%$ & 29 & $69,0 \%$ & 28 & $66,7 \%$ & 30 & $71,4 \%$ \\
\hline TSD igual a $28 \%$ & 29 & $69,0 \%$ & 28 & $66,7 \%$ & 30 & $71,4 \%$ \\
\hline TSD igual a $29 \%$ & 29 & $69,0 \%$ & 28 & $66,7 \%$ & 30 & $71,4 \%$ \\
\hline TSD igual a $30 \%$ & 29 & $69,0 \%$ & 28 & $66,7 \%$ & 30 & $71,4 \%$ \\
\hline TSD igual a $40 \%$ & 32 & $76,2 \%$ & 28 & $66,7 \%$ & 30 & $71,4 \%$ \\
\hline TSD igual a 50\% & 32 & $76,2 \%$ & 30 & $71,4 \%$ & 30 & $71,4 \%$ \\
\hline TSD igual a $100 \%$ & 33 & $78,6 \%$ & 30 & $71,4 \%$ & 33 & $78,6 \%$ \\
\hline TSD igual a $150 \%$ & 33 & $78,6 \%$ & 33 & $78,6 \%$ & 33 & $78,6 \%$ \\
\hline TSD igual a $200 \%$ & 33 & $78,6 \%$ & 33 & $78,6 \%$ & 33 & $78,6 \%$ \\
\hline TSD igual a $300 \%$ & 34 & $81,0 \%$ & 33 & $78,6 \%$ & 33 & $78,6 \%$ \\
\hline TSD igual a $400 \%$ & 34 & $81,0 \%$ & 33 & $78,6 \%$ & 34 & $81,0 \%$ \\
\hline TSD igual a $500 \%$ & 34 & $81,0 \%$ & 33 & $78,6 \%$ & 34 & $81,0 \%$ \\
\hline TSD mayor a $500 \%$ & 42 & $100,0 \%$ & 42 & $100,0 \%$ & 42 & $100,0 \%$ \\
\hline
\end{tabular}

Rev. Est. de Políticas Públicas, 5(2): julio 2019 - noviembre 2019 
Anexo 3: Valores TDLA y criterio costo de referencia

\begin{tabular}{|c|c|c|c|c|c|c|c|c|c|c|}
\hline \multicolumn{11}{|c|}{ RESUMEN TDLA PROYECTOS APR FACTOR EXPONENCIAL E HIPERBÓLICOS } \\
\hline $\begin{array}{c}\mathrm{N}^{\circ} \\
\text { Proyecto }\end{array}$ & $\begin{array}{c}\text { TDLA } \\
\text { Exponencial }\end{array}$ & \begin{tabular}{c|} 
TDLA \\
Hiperbólico \\
un parámetro
\end{tabular} & \begin{tabular}{|c|} 
TDLA \\
Hiperbólico \\
ge neralizado
\end{tabular} & $\begin{array}{c}\text { Inversión } \\
\text { UF }\end{array}$ & Año & $\begin{array}{c}\mathrm{N}^{\circ} \\
\text { Arranques }\end{array}$ & $\begin{array}{c}\mathrm{N}^{\circ} \\
\text { Beneficiarios }\end{array}$ & \begin{tabular}{|c|} 
Costo \\
imversión \\
arranque
\end{tabular} & $\begin{array}{c}\text { Costo } \\
\text { referencia }\end{array}$ & $\begin{array}{c}\text { Crite rio } \\
\text { costo de } \\
\text { referencia }\end{array}$ \\
\hline 1 & $12,782 \%$ & $23,459 \%$ & $11,729 \%$ & 147.337 & 2.015 & 970 & 5.435 & 127,6 & 144 & Acepto \\
\hline 2 & $10,069 \%$ & $16,484 \%$ & $8,242 \%$ & 19.903 & 2.014 & 162 & 810 & 103,2 & 130 & Acepto \\
\hline 3 & $12,606 \%$ & $22,449 \%$ & $11,225 \%$ & 37.644 & 2.014 & 270 & 1.345 & 117,2 & 130 & Acepto \\
\hline 4 & & & & 12.902 & 2.015 & 73 & 415 & 148,5 & 144 & Rechazo \\
\hline 5 & $37,594 \%$ & $44,055 \%$ & $22,028 \%$ & 24.672 & 2.016 & 108 & 413 & 192,0 & 144 & Rechazo \\
\hline 6 & & & - & 14.430 & 2.013 & 71 & 351 & 170,8 & 130 & Rechazo \\
\hline 7 & $9,402 \%$ & $12,451 \%$ & $6,226 \%$ & 17.831 & 2.017 & 116 & 569 & 129,2 & 144 & Acepto \\
\hline 8 & $-4,460 \%$ & $-3,279 \%$ & $-1,640 \%$ & 17.874 & 2.012 & 178 & 880 & 84,4 & 130 & Acepto \\
\hline 9 & $-3,405 \%$ & $-2,596 \%$ & $-1,298 \%$ & 28.740 & 2.016 & 267 & 1.147 & 90,5 & 144 & Acepto \\
\hline 10 & & & - & 18.217 & 2.015 & 104 & 520 & 147,2 & 144 & Rechazo \\
\hline 11 & $-2,598 \%$ & $-2,187 \%$ & $-1,094 \%$ & 17.813 & 2.017 & 131 & 653 & 114,3 & 144 & Acepto \\
\hline 12 & $8,549 \%$ & $13,395 \%$ & $6,697 \%$ & 33.974 & 2.015 & 223 & 1.825 & 128,0 & 144 & Acepto \\
\hline 13 & $1,265 \%$ & $1,367 \%$ & $0,683 \%$ & 15.461 & 2.012 & 128 & 460 & 101,5 & 130 & Acepto \\
\hline 14 & $0,152 \%$ & $0,154 \%$ & $0,077 \%$ & 45.054 & 2.015 & 314 & 1.565 & 120,6 & 144 & Acepto \\
\hline 15 & & & - & 43.329 & 2.014 & 229 & 1.145 & 159,0 & 130 & Rechazo \\
\hline 16 & - & & - & 15.282 & 2.014 & 86 & 430 & 149,3 & 130 & Rechazo \\
\hline 17 & $-1,314 \%$ & $-1,205 \%$ & $-0,603 \%$ & 9.583 & 2.016 & 109 & 436 & 73,9 & 144 & Acepto \\
\hline 18 & $-3,103 \%$ & $-2,514 \%$ & $-1,257 \%$ & 15.100 & 2.014 & 258 & 640 & 49,2 & 130 & Acepto \\
\hline 19 & & & & 25.644 & 2.014 & 164 & 832 & 131,4 & 130 & Rechazo \\
\hline 20 & $210,193 \%$ & $713,611 \%$ & $356,805 \%$ & 10.385 & 2.013 & 69 & 345 & 126,5 & 130 & Acepto \\
\hline 21 & $-5,924 \%$ & $-3,883 \%$ & $-3,492 \%$ & 16.236 & 2.016 & 183 & 732 & 74,6 & 144 & Acepto \\
\hline 22 & $14,273 \%$ & $26,682 \%$ & $13,341 \%$ & 22.766 & 2.015 & 159 & 795 & 120,3 & 144 & Acepto \\
\hline 23 & $-5,486 \%$ & $-3,732 \%$ & $-1,866 \%$ & 34.984 & 2.013 & 307 & 1.270 & 95,8 & 130 & Acepto \\
\hline 24 & $-1,150 \%$ & $-1,067 \%$ & $-0,533 \%$ & 50.900 & 2.013 & 497 & 2.382 & 86,1 & 130 & Acepto \\
\hline 25 & $1,136 \%$ & $1,218 \%$ & $0,609 \%$ & 22.433 & 2.016 & 193 & 538 & 97,7 & 144 & Acepto \\
\hline 26 & $11,132 \%$ & $14,690 \%$ & $7,345 \%$ & 19.641 & 2.017 & 121 & 605 & 136,4 & 144 & Acepto \\
\hline 27 & $13,753 \%$ & $26,277 \%$ & $13,139 \%$ & 21.267 & 2.017 & 140 & 417 & 127,7 & 144 & Acepto \\
\hline 28 & $-3,281 \%$ & $-2,619 \%$ & $-1,310 \%$ & 43.857 & 2.016 & 359 & 1.233 & 102,7 & 144 & Acepto \\
\hline 29 & $-8,025 \%$ & $-4,301 \%$ & $-2,439 \%$ & 24.326 & 2.016 & 1.448 & 6.323 & 14,1 & 144 & Acepto \\
\hline 30 & $8,235 \%$ & $12,578 \%$ & $6,289 \%$ & 12.519 & 2.016 & 93 & 421 & 113,1 & 144 & Acepto \\
\hline 31 & $38,358 \%$ & $117,727 \%$ & $58,864 \%$ & 40.032 & 2.017 & 241 & 944 & 139,6 & 144 & Acepto \\
\hline 32 & $-1,044 \%$ & $-0,980 \%$ & $-0,490 \%$ & 15.900 & 2.016 & 146 & 587 & 91,5 & 144 & Acepto \\
\hline 33 & $51,643 \%$ & $147,921 \%$ & $73,961 \%$ & 30.411 & 2.014 & 210 & 1.050 & 121,7 & 130 & Acepto \\
\hline 34 & & & & 17.092 & 2.014 & 111 & 555 & 129,4 & 130 & Acepto \\
\hline 35 & $-9,196 \%$ & $-4,646 \%$ & $-2,323 \%$ & 19.878 & 2.012 & 372 & 1.735 & 44,9 & 130 & Acepto \\
\hline 36 & $19,397 \%$ & $41,298 \%$ & $20,649 \%$ & 11.953 & 2.016 & 81 & 252 & 124,0 & 144 & Acepto \\
\hline 37 & & & & 13.845 & 2.017 & 77 & 291 & 151,1 & 144 & Rechazo \\
\hline 38 & $39,601 \%$ & $111,673 \%$ & $55,836 \%$ & 20.435 & 2.015 & 125 & 610 & 137,4 & 144 & Acepto \\
\hline 39 & $10,931 \%$ & $18,441 \%$ & $9,221 \%$ & 18.585 & 2.014 & 151 & 745 & 103,4 & 130 & Acepto \\
\hline 40 & $9,501 \%$ & $15,235 \%$ & $7,617 \%$ & 13.885 & 2.014 & 117 & 585 & 99,7 & 130 & Acepto \\
\hline 41 & $1,321 \%$ & $1,433 \%$ & $0,717 \%$ & 26.965 & 2.016 & 221 & 774 & 102,5 & 144 & Acepto \\
\hline 42 & $2,288 \%$ & $2,624 \%$ & $1,312 \%$ & 12.897 & 2.013 & 130 & 650 & 83,4 & 130 & Acepto \\
\hline OBS. 1 & $\begin{array}{l}\text { AZUL ind } \\
\text { ROJO ind }\end{array}$ & royectos q & $\begin{array}{l}\text { se aprueb } \\
\text { O se aprue }\end{array}$ & al conside & dar un & desct & al $6 \%$. & & & \\
\hline OBS. 2 & $\begin{array}{l}\text { Proyecto } 21 ; \text { co } \\
\text { ihustración se reg }\end{array}$ & $\begin{array}{l}\text { fon factor de de } \\
\text { gistra el valor má }\end{array}$ & $\begin{array}{l}\text { lescuento Hiperb } \\
\text { ás alto. }\end{array}$ & ólico de un & Paráme & etro, registral & m más de una & tasa de des & iento. P & a efectos de \\
\hline OBS. 3 & $\begin{array}{l}\text { Proyectos 21, } 3 \\
\text { ihustración se reg }\end{array}$ & $\begin{array}{l}30 \text { y } 36 \text {; con fac } \\
\text { gistra el valor mé }\end{array}$ & $\begin{array}{l}\text { ctor de descuent } \\
\text { és alto. }\end{array}$ & to Hiperbólice & o Gener & ralizado, regi: & istran más de una & a tasa de de & lescuento. Pat & a efectos de \\
\hline OBS. 4 & Proyectos 4 & (1) & y 37 ; no s. & egistra valor & tasd we & desclento, to & oda ver su valor & es muy aho & tiende a & \\
\hline OBS. 5 & $\begin{array}{l}\text { El morio de la } \\
\text { asosorias }\end{array}$ & 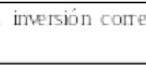 & ande a & de la ir & 8 & al de la & huación ecr & ica que $\mathrm{n}$ & incluye & zalor de las \\
\hline OBS. 6 & $\begin{array}{l}\text { La cantidad de } \\
\text { proyecto. }\end{array}$ & ananques con & esponde al nime & ero asociado & a 1 a eva & ahacion ecol & monica y los be & neficianos a & a ta puesta el & marcha del \\
\hline OBS. 7 & $\begin{array}{l}\text { Se adjunta cohur } \\
\text { del proyecto cor }\end{array}$ & mana que indic & ultado al a & elcriterio & ${ }^{\prime} \mathrm{Co}$ & Refere & cia", que con & elco: & e in & manque \\
\hline
\end{tabular}


Anexo 4: Variación en la TAP

\begin{tabular}{|c|c|c|c|c|c|c|c|c|c|}
\hline \multirow{3}{*}{ TSD } & \multicolumn{3}{|c|}{ CASO 1} & \multicolumn{3}{|c|}{ CASO 2} & \multicolumn{3}{|c|}{ CASO 3} \\
\hline & \multicolumn{3}{|c|}{$\begin{array}{l}\text { Incremento inversión inicial } \\
\text { Item } 7 \% \text { costos de distribución }\end{array}$} & \multicolumn{3}{|c|}{\begin{tabular}{|l|} 
Incremento inversión reposición \\
Item $25 \%$ costos de reposición
\end{tabular}} & \multicolumn{3}{|c|}{ Incremento ambs Items } \\
\hline & $\mathbf{E}$ & $\mathrm{H}-1 \mathrm{P}$ & H - G & $\mathbf{E}$ & H - 1P & H - G & $\mathbf{E}$ & $\mathrm{H}-1 \mathrm{P}$ & H-G \\
\hline $0 \%$ & 0 & 0 & 0 & -3 & -3 & -3 & -5 & -6 & -4 \\
\hline $1 \%$ & -1 & -1 & -4 & -2 & -2 & -4 & -3 & -4 & -5 \\
\hline $2 \%$ & -4 & -4 & 0 & -4 & -4 & -4 & -5 & -6 & -5 \\
\hline $3 \%$ & 0 & -3 & $\mathbf{0}$ & -4 & -5 & -1 & -5 & -6 & -5 \\
\hline $4 \%$ & 0 & $\mathbf{0}$ & $\mathbf{0}$ & -2 & -4 & 0 & -5 & -5 & -1 \\
\hline $5 \%$ & 0 & 0 & 0 & -1 & -2 & 0 & -4 & -5 & 0 \\
\hline $6 \%$ & 0 & $\mathbf{0}$ & $\mathbf{0}$ & 0 & -1 & $\mathbf{0}$ & -1 & -5 & $\mathbf{0}$ \\
\hline $7 \%$ & $\mathbf{0}$ & $\mathbf{0}$ & -3 & $\mathbf{0}$ & -1 & -3 & $\mathbf{0}$ & -4 & -3 \\
\hline $8 \%$ & 0 & 0 & -3 & 0 & 0 & -5 & 0 & -1 & -5 \\
\hline $9 \%$ & -2 & $\mathbf{0}$ & -4 & -2 & $\mathbf{0}$ & -5 & -2 & $\mathbf{0}$ & -6 \\
\hline $10 \%$ & -3 & $\mathbf{0}$ & -3 & -4 & $\mathbf{0}$ & -5 & -4 & $\mathbf{0}$ & -6 \\
\hline $11 \%$ & -4 & $\mathbf{0}$ & -3 & -6 & $\mathbf{0}$ & -3 & -6 & $\mathbf{0}$ & -5 \\
\hline $12 \%$ & -4 & $\mathbf{0}$ & -5 & -4 & $\mathbf{0}$ & -5 & -7 & $\mathbf{0}$ & -7 \\
\hline $13 \%$ & -6 & -2 & -4 & -6 & -2 & -3 & -8 & -2 & -6 \\
\hline $14 \%$ & -5 & -3 & -5 & -4 & -3 & -5 & -8 & -3 & -8 \\
\hline $15 \%$ & -5 & -3 & -5 & -4 & -4 & -4 & -9 & -4 & -7 \\
\hline $16 \%$ & -5 & -3 & -5 & -3 & -5 & -4 & -7 & -5 & -7 \\
\hline $17 \%$ & -5 & -4 & -5 & -2 & -5 & -2 & -7 & -6 & -7 \\
\hline $18 \%$ & -4 & -4 & -4 & -1 & -5 & -2 & -6 & -6 & -7 \\
\hline $19 \%$ & -4 & -4 & -4 & 0 & -5 & -1 & -5 & -6 & -6 \\
\hline $20 \%$ & -4 & -3 & -4 & -1 & -5 & 0 & -6 & -6 & -5 \\
\hline $21 \%$ & -4 & -3 & -4 & -1 & -4 & -1 & -5 & -6 & -6 \\
\hline $22 \%$ & -3 & -3 & -4 & -1 & -3 & 0 & -5 & -5 & -6 \\
\hline $23 \%$ & -2 & -4 & -5 & 0 & -4 & -1 & -5 & -6 & -7 \\
\hline $24 \%$ & -2 & -5 & -5 & 0 & -5 & -1 & -4 & -7 & -6 \\
\hline $25 \%$ & -2 & -4 & -4 & 0 & -4 & -1 & -3 & -6 & -6 \\
\hline $26 \%$ & -2 & -4 & -3 & 0 & -3 & 0 & -3 & -6 & -5 \\
\hline $27 \%$ & -1 & -5 & -2 & 0 & -5 & 0 & -2 & -8 & -5 \\
\hline $28 \%$ & 0 & -5 & -2 & 0 & -5 & 0 & -2 & -8 & -5 \\
\hline $29 \%$ & 0 & -5 & -2 & 0 & -4 & 0 & -1 & -8 & -5 \\
\hline $30 \%$ & 0 & -5 & -2 & 0 & -4 & 0 & -1 & -7 & -4 \\
\hline $40 \%$ & -3 & -4 & 0 & -2 & 0 & 0 & -3 & -5 & -1 \\
\hline $50 \%$ & -2 & -4 & 0 & 0 & -1 & 0 & -2 & -6 & 0 \\
\hline $100 \%$ & -3 & 0 & -3 & 0 & 0 & 0 & -3 & 0 & -3 \\
\hline $150 \%$ & -3 & -3 & -3 & 0 & -2 & 0 & -3 & -3 & -3 \\
\hline $200 \%$ & -3 & -3 & -3 & 0 & 0 & 0 & -3 & -3 & -3 \\
\hline $300 \%$ & -4 & -3 & -3 & 0 & 0 & 0 & -4 & -3 & -3 \\
\hline $400 \%$ & -4 & -3 & -4 & 0 & 0 & -1 & -4 & -3 & -4 \\
\hline $500 \%$ & -4 & -3 & -4 & 0 & 0 & 0 & -4 & -3 & -4 \\
\hline$>500 \%$ & 0 & 0 & 0 & 0 & 0 & 0 & 0 & 0 & 0 \\
\hline
\end{tabular}

Rev. Est. de Políticas Públicas, 5(2): julio 2019 - noviembre 2019 
Anexo 5: Variación en la TAP caso $3(\mathrm{~N})$

\begin{tabular}{|c|c|c|c|c|c|c|}
\hline \multirow[t]{2}{*}{ TSD } & \multicolumn{3}{|c|}{$\begin{array}{c}\text { CASO } 3 \\
\text { Costo de Referee ncia } \\
130 \text { y } 144 \text { UF por Arranque }\end{array}$} & \multicolumn{3}{|c|}{$\begin{array}{c}\text { CASO } 3 \text { (N) } \\
\text { Costo de Referencia } \\
141 \text { y } 152 \text { UF por Arranque }\end{array}$} \\
\hline & $\mathbf{E}$ & H - 1P & H - G & $E$ & H - 1P & H-G \\
\hline $0 \%$ & -5 & -6 & -4 & -2 & -2 & 0 \\
\hline $1 \%$ & -3 & -4 & -5 & -1 & -1 & -1 \\
\hline $2 \%$ & -5 & -6 & -5 & -2 & -2 & -1 \\
\hline $3 \%$ & -5 & -6 & -5 & -3 & -3 & 1 \\
\hline $4 \%$ & -5 & -5 & -1 & 0 & -2 & 1 \\
\hline $5 \%$ & -4 & -5 & 0 & 0 & 0 & 1 \\
\hline $6 \%$ & -1 & -5 & 0 & 0 & 0 & 1 \\
\hline $7 \%$ & 0 & -4 & -3 & 0 & 0 & -2 \\
\hline $8 \%$ & 0 & -1 & -5 & 0 & 0 & -2 \\
\hline $9 \%$ & -2 & 0 & -6 & -2 & 0 & -1 \\
\hline $10 \%$ & -4 & 0 & -6 & -2 & 0 & 1 \\
\hline $11 \%$ & -6 & 0 & -5 & -1 & 0 & 1 \\
\hline $12 \%$ & -7 & 0 & -7 & 0 & 0 & -1 \\
\hline $13 \%$ & -8 & -2 & -6 & -1 & -2 & -1 \\
\hline $14 \%$ & -8 & -3 & -8 & -2 & -3 & -2 \\
\hline $15 \%$ & -9 & -4 & -7 & -2 & -3 & -2 \\
\hline $16 \%$ & -7 & -5 & -7 & -1 & -3 & -1 \\
\hline $17 \%$ & -7 & -6 & -7 & 0 & -3 & 0 \\
\hline $18 \%$ & -6 & -6 & -7 & 0 & -2 & 0 \\
\hline $19 \%$ & -5 & -6 & -6 & 0 & 0 & 0 \\
\hline $20 \%$ & -6 & -6 & -5 & 0 & 1 & 0 \\
\hline $21 \%$ & -5 & -6 & -6 & 0 & 1 & 0 \\
\hline $22 \%$ & -5 & -5 & -6 & 0 & 1 & 1 \\
\hline $23 \%$ & -5 & -6 & -7 & 0 & 0 & 0 \\
\hline $24 \%$ & -4 & -7 & -6 & 0 & -1 & 0 \\
\hline $25 \%$ & -3 & -6 & -6 & 0 & -1 & 0 \\
\hline $26 \%$ & -3 & -6 & -5 & 0 & -1 & 0 \\
\hline $27 \%$ & -2 & -8 & -5 & 0 & -3 & 0 \\
\hline $28 \%$ & -2 & -8 & -5 & 0 & -2 & 0 \\
\hline $29 \%$ & -1 & -8 & -5 & 0 & -2 & 0 \\
\hline $30 \%$ & -1 & -7 & -4 & 1 & -2 & 0 \\
\hline $40 \%$ & -3 & -5 & -1 & 1 & 0 & 0 \\
\hline $50 \%$ & -2 & -6 & 0 & 1 & 0 & 1 \\
\hline $100 \%$ & -3 & 0 & -3 & 1 & 1 & 1 \\
\hline $150 \%$ & -3 & -3 & -3 & 1 & 0 & 1 \\
\hline $200 \%$ & -3 & -3 & -3 & 1 & 1 & 1 \\
\hline $300 \%$ & -4 & -3 & -3 & 1 & 1 & 1 \\
\hline $400 \%$ & -4 & -3 & -4 & 1 & 1 & 0 \\
\hline $500 \%$ & -4 & -3 & -4 & 1 & 1 & 1 \\
\hline$>500 \%$ & 0 & 0 & 0 & 0 & 0 & 0 \\
\hline
\end{tabular}

(E): Exponencial; (H - 1P): Hiperbólico un paráme tro; (H - G): Hiperbólico generalizado 


\section{Anexo 6}

\begin{tabular}{|cl|}
\hline & \multicolumn{1}{c|}{ LISTA PRINCIPALES ABREVIATURAS } \\
\hline APR & Agua Potable Rural \\
BID & Banco Interamericano de Desarrollo \\
CAE & Costo Anual Equivalente \\
CASEN & Encuesta de Caracterización Socioeconómica Nacional \\
COSC & Costo de Oportunidad Social del Capital \\
DGA & Dirección General de Aguas \\
DOH & Dirección de Obras Hidráulicas \\
FCS & Factor de Corrección Social \\
INE & Instituto Nacional de Estadística \\
MDS & Ministerio de Desarrollo Social \\
MOP & Ministerio de Obras Públicas \\
RS & Aprobación sin condiciones a las iniciativas de inversión que se presenten al \\
& Sistema Nacional de Inversión \\
TAP & Tasa de Aprobación de Proyectos \\
TIR & Tasa Interna de Retorno \\
TSD & Tasa Social de Descuento \\
TSPI & Tasa Social de Preferencia Intertemporal \\
TDLA & Tasa de Descuento Límite de Aprobación \\
UF & Unidad de Fomento \\
VABN & Valor Actual Beneficios Sociales Netos \\
VAC & Valor Actual de los Costos \\
VAN & Valor Actual Beneficios Neto \\
\hline
\end{tabular}

Rev. Est. de Políticas Públicas, 5(2): julio 2019 - noviembre 2019 